\title{
ON BLOCK SEQUENCES OF STEINER QUADRUPLE SYSTEMS WITH ERROR CORRECTING CONSECUTIVE UNIONS*
}

\author{
GENNIAN $\mathrm{GE}^{\dagger}$, YING MIAO ${ }^{\ddagger}$ AND XIANDE $\mathrm{ZHANG}^{\dagger}$ \\ Dedicated to Professor Sanpei Kageyama on the occasion of his retirement from Hiroshima \\ University
}

\begin{abstract}
Motivated by applications in combinatorial group testing for consecutive positives, we investigate a block sequence of a maximum packing $\operatorname{MP}(t, k, v)$ which contains the blocks exactly once such that the collection of all blocks together with all unions of two consecutive blocks of this sequence forms an error correcting code with minimum distance $d$. Such a sequence is usually called a block sequence with consecutive unions having minimum distance $d$, and denoted by $\operatorname{BSCU}(t, k, v \mid d)$. In this paper, we show that the necessary conditions for the existence of $\operatorname{BSCU}(3,4, v \mid 4) \mathrm{s}$ of Steiner quadruple systems, namely, $v \equiv 2,4(\bmod 6)$ and $v \geq 4$, are also sufficient, excepting $v=8,10$.
\end{abstract}

Key words. BSCU, CSCU, CSCU-CQS, CSCU-GDD

AMS subject classifications. 92D20, 94C12, 05B05

DOI. $10.1137 / 08071750 \mathrm{X}$

1. Introduction. Let $V$ be a finite set of $v$ elements and let $\mathcal{X}$ be a collection of $k$-subsets of $V$ with $|\mathcal{X}|=m$. Let $S=\left[x_{0}, x_{1}, \ldots, x_{m-1}\right]$ be a sequence of the elements in $\mathcal{X}$. The indices of the elements $x_{i}$ of $S$ are considered modulo $m$. Define $y_{i}=x_{i} \cup x_{i+1}$ for each $i, 0 \leq i \leq m-1$. The sequence $S$ is called a cyclic sequence of $\mathcal{X}$ with consecutive unions having minimum distance $d$, denoted as $\operatorname{CSCU}(k, v \mid d)$, if $C=\left\{x_{0}, \ldots, x_{m-1}, y_{0}, \ldots, y_{m-1}\right\}$ has minimum distance $d$. Note that the distance between any two sets $x$ and $y$ is defined as $d(x, y)=|(x \cup y) \backslash(x \cap y)|$. Furthermore, a $\operatorname{CSCU}(k, v \mid d)$ is said to be maximal if the number of elements in $\mathcal{X}$ is maximum for given $k, v$, and $d$, denoted as $\operatorname{MCSCU}(k, v \mid d)$.

The concept of an MCSCU is motivated by the applications in combinatorial group testing for consecutive positives. Group testing was proposed by Dorfman [3] in 1940s to do large scale blood testing economically, and new applications of group testing have been found recently such as DNA library screening, being error-prone, in which it is desired to determine the set of all clones containing a specific sequence of nucleotides in an economical and correct way. A clone is positive if it contains the specific sequence and negative otherwise. One chooses arbitrarily a subset of clones called a group or pool and tests all clones in the pool in one stroke by some chemical analysis. The pool is positive when it contains at least one positive clone and negative otherwise. Colbourn [1] developed some strategy for group testing when the clones are linearly

* Received by the editors March 3, 2008; accepted for publication (in revised form) February 11, 2009; published electronically May 20, 2009.

http://www.siam.org/journals/sidma/23-2/71750.html

${ }^{\dagger}$ Department of Mathematics, Zhejiang University, Hangzhou 310027, Zhejiang, People's Republic of China (gnge@zju.edu.cn). The first author's research was supported by the National Outstanding Youth Science Foundation of China under grant 10825103, National Natural Science Foundation of China under grant 10771193, Specialized Research Fund for the Doctoral Program of Higher Education, Program for New Century Excellent Talents in University, and Zhejiang Provincial Natural Science Foundation of China under grant D7080064.

${ }^{\ddagger}$ Department of Social Systems and Management, Graduate School of Systems and Information Engineering, University of Tsukuba, Tsukuba, Ibaraki 305-8573, Japan (miao@sk.tsukuba.ac.jp). This author's research was supported by Grant-in-Aid for Scientific Research (C) under grant 18540109 . 
ordered, and the positive clones form a consecutive subset of the set of all clones, the typical example being the problem of locating a sequence-tagged site (or STS) among ordered clones. Jimbo and his collaborators [16, 15, 17, 18] improved Colbourn's strategy by considering the error detecting and correcting capability of group testing which is essential in view of applications such as DNA library screening. In particular, Momihara and Jimbo [16, 15] suggested using MCSCUs of a combinatorial structure called t-packings to correct false negative or false positive clones in the pool outcomes. For more details of such applications, we refer the reader to $[1,4,16,15,17,18,19]$ and references therein.

A $t$-packing of order $v$, block size $k$, briefly $\mathrm{P}(t, k, v)$, is an ordered pair $(V, \mathcal{B})$, where $V$ is a finite set of $v$ elements called points, and $\mathcal{B}$ is a set of $k$-subsets of $V$ called blocks, such that each $t$-tuple of distinct points of $V$ is contained in at most one block of $\mathcal{B}$. In particular, a $\mathrm{P}(t, k, v)$ is said to be maximal, denoted $\mathrm{MP}(t, k, v)$, if the number of blocks is maximum for given $t, k$, and $v$. For $v \equiv 2,4(\bmod 6)$, an $\operatorname{MP}(3,4, v)$ is also called a Steiner quadruple system, briefly $\operatorname{SQS}(v)$. The existence of an $\operatorname{SQS}(v)$ for every admissible $v$ is proved by Hanani [5], Hartman [7, 8], and Lenz $[12]$.

It is known (see [16]) that a $\operatorname{CSCU}(k, v \mid d)$ of $\mathcal{B}$ is maximal if $\mathcal{B}$ is the block set of an $\operatorname{MP}(\lfloor k-d / 2\rfloor+1, k, v)$. $\operatorname{A~CSCU}(k, v \mid d)$ of $\mathcal{B}$ which is the block set of an $\operatorname{MP}(t, k, v)$ is also called a block sequence of $\mathcal{B}$ with consecutive unions having minimum distance $d$, briefly $\operatorname{BSCU}(t, k, v \mid d)$.

In the case of $d=2$, Müller and Jimbo [18] showed that there exists a $\operatorname{BSCU}(k, k, v$ $\mid 2)$ for every $v \geq v_{k}$ for the pairs of parameters $k$ and $v_{k},\left(k, v_{k}\right)=(2,6),(3,8),(4,11)$, $(5,12),(6,17)$, and $(7,19)$, without introducing the notion of block sequences of $t$ packings. In the case of $d=3$, Momihara and Jimbo [16] showed the existence of a $\operatorname{BSCU}(2,3, v \mid 3)$ for every $v \geq 10$. For the case of $d=4$, it is clear that a $\operatorname{BSCU}(3,4, v \mid 4)$ forms an $\operatorname{MCSCU}(4, v \mid 4)$. Momihara and Jimbo [15] recently showed the existence of a $\operatorname{BSCU}(3,4, v \mid 4)$ for 47 small values $v \leq 500$ using the following two constructions.

THEOREM 1.1. (see [15]). Let $v$ be an integer satisfying $v \equiv 2,4(\bmod 6)$ and $v \geq 14$.

(1) If there exists a $\mathrm{BSCU}(3,4, v \mid 4)$, then there exists a $\operatorname{BSCU}(3,4,2 v \mid 4)$ which contains a sub-BSCU $(3,4, v \mid 4)$.

(2) If there exists a $\operatorname{BSCU}(3,4, v \mid 4)$, then there exists a $\operatorname{BSCU}(3,4,3 v-2 \mid 4)$ which contains a sub-BSCU $(3,4, v \mid 4)$.

It is not difficult to see [15] that if there exists a $\operatorname{BSCU}(3,4, v \mid 4)$, then every two consecutive blocks must be disjoint. Furthermore, there does not exist a $\operatorname{BSCU}(3,4, v \mid 4)$ for $v \leq 11$ except for $v=4$, in which there is only one block. We call such a $\operatorname{BSCU}(3,4,4 \mid 4)$ trivial.

In this paper, we write $\operatorname{BSCU}(3,4, v \mid 4)$ of the block sets of Steiner quadruple systems as $\operatorname{BSCU}(v)$ for brevity. The necessary conditions for the existence of a $\operatorname{BSCU}(v)$ are $v \equiv 2,4(\bmod 6)$ and $v \geq 4$. In the following sections, we will prove that the above necessary conditions are also sufficient except for $v=8,10$. Our main tools are the recursive constructions used in the 3-design theory (see $[9,10,11]$ for detailed information).

2. Recursive constructions. Let $v, s$ be two nonnegative integers, $t$ be a positive integer, and $K$ be a set of positive integers. A candelabra t-system (or $t$-CS) of order $v$ and block sizes from $K$, denoted by $\operatorname{CS}(t, K, v)$, is a quadruple $(X, S, \mathcal{G}, \mathcal{A})$ that satisfies the following properties: 
(1) $X$ is a set of $v$ elements (called points);

(2) $S$ is an $s$-subset (called the stem of the candelabra) of $X$;

(3) $\mathcal{G}=\left\{G_{1}, G_{2}, \ldots\right\}$ is a set of nonempty subsets (called groups or branches) of $X \backslash S$, which partition $X \backslash S$;

(4) $\mathcal{A}$ is a collection of subsets (called blocks) of $X$, each of cardinality from $K$;

(5) every $t$-subset $T$ of $X$ with $\left|T \cap\left(S \cup G_{i}\right)\right|<t$ for all $i$ is contained in a unique block of $\mathcal{A}$, and no $t$-subset of $S \cup G_{i}$ for all $i$ is contained in any block of $\mathcal{A}$.

By the group type (or type) of a $t$-CS $(X, S, \mathcal{G}, \mathcal{A})$ we mean the list $(|G| \mid G \in$ $\mathcal{G}:|S|$ ) of group sizes and stem size, where the stem size is separated from the group sizes by a colon. If a $t$-CS has $n_{i}$ groups of size $g_{i}, 1 \leq i \leq r$, and stem size $s$, then we use the notation $\left(g_{1}^{n_{1}} g_{2}^{n_{2}} \ldots g_{r}^{n_{r}}: s\right)$ to denote the group type. When $t=3$ and $K=\{4\}$, such a system is usually called a candelabra quadruple system and denoted for short by $\operatorname{CQS}\left(g_{1}^{n_{1}} g_{2}^{n_{2}} \ldots g_{r}^{n_{r}}: s\right)$.

A holey quadruple system of order $v$ with a hole of order $s$, denoted by $\operatorname{HSQS}(v: s)$, is a triple $(X, S, \mathcal{A})$ where $X$ is a set of $v$ elements (called points), $S$ is an $s$-subset of $X$, and $\mathcal{A}$ is a collection of 4 -subsets (called blocks) of $X$ such that every 3 -subset $T$ of $X$ with $T \nsubseteq S$ is contained in a unique block of $\mathcal{A}$ and no 3-subset of $S$ is contained in any block of $\mathcal{A}$.

Let $(X, S, \mathcal{G}, \mathcal{A})$ be a $\operatorname{CS}(3, K, v)$ of type $\left(g_{1}^{n_{1}} g_{2}^{n_{2}} \ldots g_{r}^{n_{r}}: s\right)$ with $S=\left\{\infty_{1}, \infty_{2}, \ldots\right.$, $\left.\infty_{s}\right\}$, where $s \geq 1$. For $1 \leq i \leq s$, let $\mathcal{B}_{i}=\left\{A \backslash\left\{\infty_{i}\right\} \mid \infty_{i} \in A \in \mathcal{A}\right\}$ and $\mathcal{T}=\{A \in \mathcal{A} \mid A \cap S=\emptyset\}$. Then the $(s+3)$-tuple $\left(X \backslash S, \mathcal{G}, \mathcal{B}_{1}, \mathcal{B}_{2}, \ldots, \mathcal{B}_{s}, \mathcal{T}\right)$ is called an $s$-fan design. If block sizes of $\mathcal{B}_{i}, 1 \leq i \leq s$, and $\mathcal{T}$ are from $K_{i}$ and $K_{\mathcal{T}}$, respectively, then the $s$-fan design is denoted by $s-\mathrm{FG}\left(3,\left(K_{1}, \ldots, K_{s}, K_{\mathcal{T}}\right), \sum_{i=1}^{r} n_{i} g_{i}\right)$ of type $g_{1}^{n_{1}} g_{2}^{n_{2}} \ldots g_{r}^{n_{r}}$.

A group divisible $t$-design of order $v$ and block sizes from $K$, denoted by $\operatorname{GDD}(t, K$, $v)$, is a triple $(X, \mathcal{G}, \mathcal{B})$ such that

(1) $X$ is a set of $v$ elements (called points);

(2) $\mathcal{G}=\left\{G_{1}, G_{2}, \ldots\right\}$ is a set of nonempty subsets (called groups) of $X$ which partition $X$;

(3) $\mathcal{B}$ is a collection of subsets (called blocks) of $X$, each of cardinality from $K$, such that each block intersects any given group in at most one point; and

(4) every $t$-subset $T$ of $X$ from $t$ distinct groups is contained in a unique block of $\mathcal{B}$.

The type of $\operatorname{GDD}(t, K, v)$ is defined as the list $(|G| \mid G \in \mathcal{G})$. In this paper, we consider only $\operatorname{GDD}(3,4, v)$ of type $T$ and always write $\operatorname{GDD}(T)$ for brevity.

Theorem 2.1. (see [14]). For $u>3, u \neq 5$, a $\operatorname{GDD}\left(g^{u}\right)$ exists if and only if $u g$ is even and $g(u-1)(u-2)$ is divisible by 3 . For $u=5$, a $\operatorname{GDD}\left(g^{5}\right)$ exists if $g$ is divisible by 4 or 6 .

A $\operatorname{CSCU}(4, v \mid 4)$ of $\mathcal{B}$ which is the block set of a $\operatorname{CQS}\left(g_{1}^{n_{1}} g_{2}^{n_{2}} \ldots g_{r}^{n_{r}}: s\right)$ will be denoted by CSCU-CQS $\left(g_{1}^{n_{1}} g_{2}^{n_{2}} \ldots g_{r}^{n_{r}}: s\right)$ in this paper. Similarly, we can define CSCU-HSQS, CSCU-GDD, etc.

Now we apply the fundamental constructions in the 3-design theory, where "filling in holes" and the "weighting method" are always useful (see [9]). First, we may think of one CSCU (the master design) as a cycle which can be cut off at any place. Next, we view the sequence of the other cut-off CSCU (the subdesign) as a segment and insert it into some cut place of the master design to form a bigger cycle. Then we calculate the number of the places in the master design where the obtained bigger cycle is also a CSCU. If this number is positive, then the construction succeeds. We explain it in detail as follows. 
For any $k$-subset sequence $S=\left[x_{0}, x_{1}, \ldots, x_{m-1}\right]$ with length $m$, define

$$
\begin{aligned}
& \sigma^{j}(S)=\left[x_{j}, x_{j+1}, \ldots, x_{m-1}, x_{0}, \ldots, x_{j-1}\right], \\
& \bar{S}=\left\{x_{0} \cup x_{1}, x_{1} \cup x_{2}, \ldots, x_{m-2} \cup x_{m-1}\right\},
\end{aligned}
$$

and

$$
\widehat{S}=\left\{x_{0} \cup x_{1}, x_{1} \cup x_{2}, \ldots, x_{m-2} \cup x_{m-1}, x_{m-1} \cup x_{0}\right\} .
$$

Let $U, V$ be two finite sets with $|U|=u$ and $|V|=v$, where $U$ is not necessarily disjoint with $V$. Let $S=\left[b_{0}, b_{1}, \ldots, b_{p-1}\right]$ be a $\operatorname{CSCU}(4, u \mid 4)$ of $\mathcal{B}$ which is a collection of 4 -subsets of $U$ with $p=|\mathcal{B}|$, and let $T=\left[a_{0}, a_{1}, \ldots, a_{q-1}\right]$ be a $\operatorname{CSCU}(4, v \mid 4)$ of $\mathcal{A}$ which is a collection of 4-subsets of $V$ with $q=|\mathcal{A}|$. It is clear that $\left|b \cap b^{\prime}\right| \leq 2$ and $\left|a \cap a^{\prime}\right| \leq 2$ for any distinct $b, b^{\prime} \in \mathcal{B}$ and $a, a^{\prime} \in \mathcal{A}$. We may assume that, for any $b \in \mathcal{B}$, we always have $|b \cap V| \leq 2$. Then, for any $a \in \mathcal{A}$ and $b \in \mathcal{B}$, we always have $|b \cap a| \leq 2$. We view $S$ as a cycle, cut $T$ between $a_{0}$ and $a_{q-1}$, and keep the order fixed. We insert $T=\left[a_{0}, a_{1}, \ldots, a_{q-1}\right]$ into $S$ between $b_{i-1}$ and $b_{i}$ for some $i, 0 \leq i \leq p-1$, and denote the bigger cycle $\left[a_{0}, a_{1}, \ldots, a_{q-1}, b_{i}, b_{i+1}, \ldots, b_{i-1}\right]$ by $S_{i}=\left[T, \sigma^{i}(S)\right]$. Let $M=\left\{i \mid S_{i}\right.$ is $\operatorname{CSCU}(4, w \mid 4)$ of $\left.\mathcal{B} \cup \mathcal{A}, 0 \leq i \leq p-1\right\}$, where $w=|U \cup V|$, and let $|M|=m$. If $m>0$, then we obtain a bigger $\operatorname{CSCU}(4, w \mid 4)$ from the two small CSCUs. Next, we estimate the value of $m$.

Let $C=\mathcal{A} \cup \mathcal{B} \cup \bar{T} \cup \overline{\sigma^{i}(S)} \cup D$, where $D=\left\{a_{0} \cup b_{i-1}, a_{q-1} \cup b_{i}\right\}$. We check the distance between any two elements of $C$. First, we consider the case that $a_{0} \cap b_{i-1}=\emptyset$ and $a_{q-1} \cap b_{i}=\emptyset$. In this case, we have the following conclusions:

Since $T$ is a $\operatorname{CSCU}$ of $\mathcal{A}$, we have

Case 1: $d\left(a, a^{\prime}\right) \geq 4$ for any $a, a^{\prime} \in \mathcal{A}$;

Case 2: $d\left(c, c^{\prime}\right) \geq 4$ for any $c, c^{\prime} \in \bar{T}$; and

Case 3: $d(a, c) \geq 4$ for any $a \in \mathcal{A}$ and $c \in \bar{T}$.

Since $S$ is a CSCU of $\mathcal{B}$, we have

Case 4: $d\left(b, b^{\prime}\right) \geq 4$ for any $b, b^{\prime} \in \mathcal{B}$;

Case 5: $d\left(c, c^{\prime}\right) \geq 4$ for any $c, c^{\prime} \in \overline{\sigma^{i}(S)}$; and

Case 6: $d(b, c) \geq 4$ for any $b \in \mathcal{B}$ and $c \in \overline{\sigma^{i}(S)}$.

Since $\left|a_{0} \cap b_{i}\right| \leq 2,\left|a_{q-1} \cap b_{i-1}\right| \leq 2$, and $b_{i-1} \cap b_{i}=\emptyset$, we know that $d\left(b_{i-1}, b_{i}\right)=8$, $d\left(a_{0} \cup b_{i-1}, b_{i}\right) \geq 6$, and also

Case 7: $d\left(a_{0} \cup b_{i-1}, a_{q-1} \cup b_{i}\right) \geq 4$.

Since $|a \cap b| \leq 2$, we have

Case 8: $d(a, b) \geq 4$ for any $a \in \mathcal{A}, b \in \mathcal{B}$.

Since $|a|=4,|b|=4$, and $|c|=8$ for any $a \in \mathcal{A}, b \in \mathcal{B}$, and $c \in \bar{T} \cup \overline{\sigma^{i}(S)} \cup D$, we have

Case 9: $d(a, c) \geq 4$ for any $a \in \mathcal{A}$ and $c \in \overline{\sigma^{i}(S)} \cup D$; and

Case 10: $d(b, c) \geq 4$ for any $b \in \mathcal{B}$ and $c \in \bar{T} \cup D$.

Since $|b \cap V| \leq 2$ for any $b \in \mathcal{B}$, we have

Case 11: $d\left(c, c^{\prime}\right) \geq 4$ for $c \in \bar{T}$ and $c^{\prime} \in \overline{\sigma^{i}(S)} \cup D$.

Under the assumption that $a_{0} \cap b_{i-1}=\emptyset$ and $a_{q-1} \cap b_{i}=\emptyset$, we still need to consider the values of $d\left(c, c^{\prime}\right)$ for any $c \in \overline{\sigma^{i}(S)}$ and $c^{\prime} \in D$. Note that we should also check the distance between any two elements of $C$ in the case that $a_{0} \cap b_{i-1} \neq \emptyset$ or $a_{q-1} \cap b_{i} \neq \emptyset$.

Let $N\left(a_{q-1}\right)=\left\{k \mid 0 \leq k \leq p-1, a_{q-1} \cap b_{k} \neq \emptyset\right.$ or $d\left(a_{q-1} \cup b_{k}, c\right)<4$ for some $c \in$ $\widehat{S}\}$ and $n\left(a_{q-1}\right)=\left|N\left(a_{q-1}\right)\right|$. Also let $\alpha\left(a_{q-1}\right)=\left|\left\{k \mid 0 \leq k \leq p-1, a_{q-1} \cap b_{k} \neq \emptyset\right\}\right|$. 
Then $n\left(a_{q-1}\right)=\alpha\left(a_{q-1}\right)+\mid\left\{k \mid 0 \leq k \leq p-1, a_{q-1} \cap b_{k}=\emptyset\right.$, and $d\left(a_{q-1} \cup\right.$ $\left.b_{k}, c\right)<4$ for some $\left.c \in \widehat{S}\right\} \mid$. In order to estimate $n\left(a_{q-1}\right)$, we consider the case that $a_{q-1} \cap b_{k}=\emptyset$. It is clear that for any index $0 \leq l \leq p-1,\left|\left(b_{l} \cup b_{l+1}\right) \cap a_{q-1}\right| \leq 4$.

If there exists an index $l$ such that $\left|\left(b_{l} \cup b_{l+1}\right) \cap a_{q-1}\right|=4$, i.e., $\left|b_{l} \cap a_{q-1}\right|=2$ and $\left|b_{l+1} \cap a_{q-1}\right|=2$, then if $d\left(b_{l} \cup b_{l+1}, a_{q-1} \cup b_{k}\right)<4$, we should have $\left|\left(b_{l} \cup b_{l+1}\right) \cap b_{k}\right| \geq 3$. In the case that $\mathcal{B}$ is the block set of some 3-packing of order $u$, there is at most one such $k$ that $\left|\left(b_{l} \cup b_{l+1}\right) \cap b_{k}\right|=4$, that is, $b_{k}=\left(b_{l} \cup b_{l+1}\right) \backslash a_{q-1}$, or there are at most 4 such $k$ that $\left|\left(b_{l} \cup b_{l+1}\right) \cap b_{k}\right|=3$, that is, $b_{k}$ are obtained by choosing any three points from the four points in $\left(b_{l} \cup b_{l+1}\right) \backslash a_{q-1}$ and the other one from the other points of $U$, which implies that $\mid\left\{k \mid 0 \leq k \leq p-1, a_{q-1} \cap b_{k}=\emptyset\right.$, and $d\left(a_{q-1} \cup b_{k}, b_{l} \cup b_{l+1}\right)<$ $4\} \mid \leq 4$.

If there exists an index $l$ such that $\left|\left(b_{l} \cup b_{l+1}\right) \cap a_{q-1}\right|=3$, i.e., $\left|b_{l} \cap a_{q-1}\right|=2$ and $\left|b_{l+1} \cap a_{q-1}\right|=1$, or $\left|b_{l} \cap a_{q-1}\right|=1$ and $\left|b_{l+1} \cap a_{q-1}\right|=2$, then we should have $\left|\left(b_{l} \cup b_{l+1}\right) \cap b_{k}\right|=4$ if $d\left(b_{l} \cup b_{l+1}, a_{q-1} \cup b_{k}\right)<4$. In the case that $\mathcal{B}$ is the block set of some 3-packing of order $u$, there is at most one $k$ such that $\left|\left(b_{l} \cup b_{l+1}\right) \cap b_{k}\right|=4$, that is, $b_{k}$ is obtained by choosing four points from $\left(b_{l} \cup b_{l+1}\right) \backslash a_{q-1}$. If there is another $k^{\prime}$ such that $\left|\left(b_{l} \cup b_{l+1}\right) \cap b_{k^{\prime}}\right|=4$, then $\left|b_{k} \cap b_{k^{\prime}}\right| \geq 3$ because $\left|\left(b_{l} \cup b_{l+1}\right) \backslash a_{q-1}\right|=5$, which is a contradiction. In this case, we have $\mid\left\{k \mid 0 \leq k \leq p-1, a_{q-1} \cap b_{k}=\right.$ $\emptyset$, and $\left.d\left(a_{q-1} \cup b_{k}, b_{l} \cup b_{l+1}\right)<4\right\} \mid \leq 1$.

If $\left|\left(b_{l} \cup b_{l+1}\right) \cap a_{q-1}\right| \leq 2$, then we can easily check that there is no $k$ such that $d\left(b_{l} \cup b_{l+1}, a_{q-1} \cup b_{k}\right)<4$, that is, $\mid\left\{k \mid 0 \leq k \leq p-1, a_{q-1} \cap b_{k}=\emptyset\right.$, and $d\left(a_{q-1} \cup\right.$ $\left.\left.b_{k}, b_{l} \cup b_{l+1}\right)<4\right\} \mid=0$.

Therefore, if we define $\gamma\left(a_{q-1}\right)=\mid\left\{l|0 \leq l \leq p-1|,\left(b_{l} \cup b_{l+1}\right) \cap a_{q-1} \mid=\right.$ $4\} \mid$, and $\delta\left(a_{q-1}\right)=\left|\left\{l|0 \leq l \leq p-1|,\left(b_{l} \cup b_{l+1}\right) \cap a_{q-1} \mid=3\right\}\right|$, then under the condition that $a_{q-1} \cap b_{k}=\emptyset$, there are at most $4 \gamma\left(a_{q-1}\right)+\delta\left(a_{q-1}\right) k$ such that $d\left(b_{l} \cup b_{l+1}, a_{q-1} \cup b_{k}\right)<4$. So we have $n\left(a_{q-1}\right) \leq \alpha\left(a_{q-1}\right)+4 \gamma\left(a_{q-1}\right)+\delta\left(a_{q-1}\right)$. From the definition of $\gamma\left(a_{q-1}\right)$, we know that the existence of one such index $l$ in $\gamma\left(a_{q-1}\right)$ would imply both $\left|b_{l} \cap a_{q-1}\right|=2$ and $\left|b_{l+1} \cap a_{q-1}\right|=2$. Also, from the definition of $\delta\left(a_{q-1}\right)$, the existence of one such index $l$ in $\delta\left(a_{q-1}\right)$ would imply $\left|b_{l} \cap a_{q-1}\right|=2$ or $\left|b_{l+1} \cap a_{q-1}\right|=2$, but not both. Keeping in mind the possibilities of occurrences of consecutive blocks in $\Xi=\left\{k|0 \leq k \leq p-1,| b_{k} \cap a_{q-1} \mid=2\right\}$ and one block in $\Xi$ followed by one block in $\left\{k|0 \leq k \leq p-1,| b_{k} \cap a_{q-1} \mid=1\right\}$, we can know that these would imply $2 \gamma\left(a_{q-1}\right)+\delta\left(a_{q-1}\right)<2 \beta\left(a_{q-1}\right)$, since $\gamma$ and $\delta$ are mutually exclusive, where $\beta\left(a_{q-1}\right)=|\Xi|$.

Similarly, we can analyze the set $N\left(a_{0}\right)=\left\{k \mid 0 \leq k \leq p-1, a_{0} \cap b_{k-1} \neq\right.$ $\emptyset$, or $d\left(a_{0} \cup b_{k-1}, c\right)<4$ for some $\left.c \in \widehat{S}\right\}$, where $n\left(a_{0}\right)=\left|N\left(a_{0}\right)\right|$.

Then from the definitions of $M, N\left(a_{0}\right)$, and $N\left(a_{q-1}\right)$, we immediately have that $M \supseteq Z_{p} \backslash\left(N\left(a_{0}\right) \cup N\left(a_{q-1}\right)\right)$ and $m \geq p-n\left(a_{0}\right)-n\left(a_{q-1}\right)+\left|N\left(a_{0}\right) \cap N\left(a_{q-1}\right)\right| \geq$ $p-n\left(a_{0}\right)-n\left(a_{q-1}\right)+|E|$, where $E \subseteq N\left(a_{0}\right) \cap N\left(a_{q-1}\right)$.

THEOREm 2.2. Suppose that there are both a CSCU-HSQS(u:v) and a BSCU(v). Then there is a $B S C U(u)$ when $u \geq 44$ and $u>v$.

Proof. Let $S=\left[b_{0}, b_{1}, \ldots, b_{p-1}\right]$ be a $\operatorname{CSCU}-\operatorname{HSQS}(u: v)$ on $U$ and $T=$ $\left[a_{0}, a_{1}, \ldots, a_{q-1}\right]$ be a $\operatorname{BSCU}(v)$ on $V$ with $V \subset U$. By the definition of an $\operatorname{HSQS}(u$ : $v$ ), we know that for any of its blocks, say, $b$, we always have $|b \cap V| \leq 2$. We view $S$ as a cycle, cut $T$ between $a_{0}$ and $a_{q-1}$, and keep the order fixed. Next, insert $T=\left[a_{0}, a_{1}, \ldots, a_{q-1}\right]$ into $S$ between $b_{i-1}$ and $b_{i}$ for some $i, 0 \leq i \leq p-1$, and denote the resultant cycle $\left[a_{0}, a_{1}, \ldots, a_{q-1}, b_{i}, b_{i+1}, \ldots, b_{i-1}\right]$ by $S_{i}=\left[T, \sigma^{i}(S)\right]$. Using the same notation as above, we prove the theorem as follows. 
Since $T$ is a $\operatorname{BSCU}(v)$, we have $a_{q-1} \cap a_{0}=\emptyset$ since they are consecutive. From the balanced property of $t$-designs, we also have $n\left(a_{0}\right)=n\left(a_{q-1}\right)$. Then $m \geq p-$ $n\left(a_{0}\right)-n\left(a_{q-1}\right) \geq p-2\left(\alpha\left(a_{q-1}\right)+4 \gamma\left(a_{q-1}\right)+\delta\left(a_{q-1}\right)\right)>p-2 \alpha\left(a_{q-1}\right)-8 \beta\left(a_{q-1}\right)$. Here $p=u(u-1)(u-2) / 24-v(v-1)(v-2) / 24, \alpha\left(a_{q-1}\right)=2(u-1)(u-2) / 3-$ $3(u-2)-2(v-1)(v-2) / 3+3(v-2)$, and $\beta\left(a_{q-1}\right)=3(u-v)$. Then we have $m>p-2 \alpha\left(a_{q-1}\right)-8 \beta\left(a_{q-1}\right)>0$ when $u \geq 44$ and $u>v$. This means that there is a $\operatorname{BSCU}(u)$ when $u \geq 44$ and $u>v$.

THEOREM 2.3. Suppose that there are both a CSCU-CQS( $\left.m^{n}: s\right)$ and a CSCU$H S Q S(m+s: s)$. Then there are both a CSCU-HSQS(mn+s:m+s) and a CSCU$H S Q S(m n+s: s)$ when $m n \geq 44, m+2 s \geq 5$, and $m \geq 2$.

Proof. Let $\left(X, S,\left\{G_{1}, \ldots, G_{n}\right\}, \mathcal{B}\right)$ be the $\operatorname{CQS}\left(m^{n}: s\right)$. Then we construct an $\operatorname{HSQS}(m+s: s)$ on $S \cup G_{k}, 1 \leq k \leq n$, with $S$ as the hole to obtain the desired HSQS $(m n+s: m+s)$ (or HSQS $(m n+s: s)$, respectively). Let $S_{0}=\left[b_{0}, b_{1}, \ldots, b_{p-1}\right]$ be a CSCU-CQS $\left(m^{n}: s\right)$ and $T_{k}=\left[a_{0}^{k}, a_{1}^{k}, \ldots, a_{q-1}^{k}\right]$ be a CSCU-HSQS $(m+s: s)$ on $S \cup G_{k}$. Note that for each block $b \in \mathcal{B}$, we have $\left|b \cap\left(S \cup G_{k}\right)\right| \leq 2$. View $S_{0}$ as a cycle, and cut each $T_{k}$ between $a_{0}^{k}$ and $a_{q-1}^{k}$. Then we insert each $T_{k}$ into $S_{0}$ between $b_{i_{k}-1}$ and $b_{i_{k}}$ one by one. Here, we require that $i_{k} \neq i_{k^{\prime}}$ if $k \neq k^{\prime}$.

Using the same notation, we have that $p=m^{2} n(n-1)(m+m n+3 s-3) / 24$. By counting the number $r_{x}$ of blocks in $\mathcal{B}$ containing a point $x \in X$, and the assumption that $m+2 s \geq 5$, we know that $r_{x} \leq m(n-1)(m n+m+2 s-3) / 6$. By counting the number $r_{x, y}$ of blocks in $\mathcal{B}$ containing a pair of distinct points $\{x, y\}$ of $X$, and the assumption that $m \geq 2$, we also know that $r_{x, y} \geq m(n-1) / 2$. Then we have $\max \left\{\alpha\left(a_{0}^{k}\right), \alpha\left(a_{q-1}^{k}\right)\right\} \leq \alpha=4 \times m(n-1)(m n+m+2 s-3) / 6-6 \times m(n-1) / 2$ and $\max \left\{\beta\left(a_{0}^{k}\right), \beta\left(a_{q-1}^{k}\right)\right\} \leq \beta=6 \times m(n-1) / 2$ for any $1 \leq k \leq n$.

First, since $m_{1} \geq p-\alpha\left(a_{0}^{1}\right)-4 \beta\left(a_{0}^{1}\right)-\alpha\left(a_{q-1}^{1}\right)-4 \beta\left(a_{q-1}^{1}\right) \geq p-2 \alpha-8 \beta \geq 1$, there exists one $i_{1}, 0 \leq i_{1} \leq p-1$, such that $S_{1}=\left[\ldots, b_{i_{1}-1}, T_{1}, b_{i_{1}}, \ldots\right]$ is a CSCU. Here, $S_{1}$ is obtained by inserting $T_{1}$ into $S_{0}$ between $b_{i_{1}-1}$ and $b_{i_{1}}$.

Next, we want to insert $T_{2}$ into $S_{1}$ between $b_{i_{2}-1}$ and $b_{i_{2}}$, where $0 \leq i_{2} \leq p-1$ and $i_{2} \neq i_{1}$, so that $S_{2}=\left[\ldots, b_{i_{1}-1}, T_{1}, b_{i_{1}}, \ldots, b_{i_{2}-1}, T_{2}, b_{i_{2}}, \ldots\right]$ is a CSCU. Since $\left|b \cap\left(S \cup G_{2}\right)\right| \leq 2$ for each block $b \in T_{1} \cup \mathcal{B}$, in order to estimate $m_{2}$, the number of suitable places that we can properly insert $T_{2}$ into $S_{1}$, we need only to compute the numbers of the consecutive unions $c \in \widehat{S_{1}}=\overline{\sigma^{i_{1}}\left(S_{0}\right)} \cup\left\{a_{q-1}^{1} \cup b_{i_{1}}, a_{0}^{1} \cup b_{i_{1}-1}\right\} \cup \overline{T_{1}}$ such that $\left|c \cap a_{j}^{2}\right|=3$ and $4, j=0, q-1$, respectively, for the reason that $m_{2} \geq p^{\prime}-n^{\prime}\left(a_{0}^{2}\right)-$ $n^{\prime}\left(a_{q-1}^{2}\right) \geq p-1-\left(\alpha^{\prime}\left(a_{0}^{2}\right)+4 \gamma^{\prime}\left(a_{0}^{2}\right)+\delta^{\prime}\left(a_{0}^{2}\right)\right)-\left(\alpha^{\prime}\left(a_{q-1}^{2}\right)+4 \gamma^{\prime}\left(a_{q-1}^{2}\right)+\delta^{\prime}\left(a_{q-1}^{2}\right)\right)$, where $\alpha^{\prime}\left(a_{j}^{2}\right)=\left|\left\{k \mid 0 \leq k \leq p-1, b_{k} \cap a_{j}^{2} \neq \emptyset\right\}\right|=\alpha\left(a_{j}^{2}\right), \gamma^{\prime}\left(a_{j}^{2}\right)=\left|\left\{l||\left(c_{l} \cup c_{l+1}\right) \cap a_{j}^{2} \mid=4\right\}\right|$, $\delta^{\prime}\left(a_{j}^{2}\right)=\left|\left\{l||\left(c_{l} \cup c_{l+1}\right) \cap a_{j}^{2} \mid=3\right\}\right|$ for $j=0$ and $q-1$, and $c_{l} \cup c_{l+1} \in \widehat{S_{1}}$. It is easy to know that there are no such unions in $\overline{T_{1}}$. We then consider the unions in $\left\{a_{q-1}^{1} \cup b_{i_{1}}, a_{0}^{1} \cup b_{i_{1}-1}\right\} \cup \overline{\sigma^{i_{1}}\left(S_{0}\right)}$. For the unions in $\overline{\sigma^{i_{1}}\left(S_{0}\right)}$, we know that $4 \gamma\left(a_{j}^{2}\right)+$ $\delta\left(a_{j}^{2}\right)<4 \beta\left(a_{j}^{2}\right)$ holds for $j=0, q-1$. For the unions in $\left\{a_{q-1}^{1} \cup b_{i_{1}}, a_{0}^{1} \cup b_{i_{1}-1}\right\}$, since $\left|\left(a_{0}^{1} \cup a_{q-1}^{1}\right) \cap a_{j}^{2}\right| \leq 2$ for $j=0, q-1$, and $a_{0}^{1} \cap a_{q-1}^{1}=\emptyset$, we know that the only possible cases are the following: (1) both $a_{q-1}^{1} \cup b_{i_{1}}$ and $a_{0}^{1} \cup b_{i_{1}-1}$ intersect $a_{j}^{2}$ at 3 elements; (2) exactly one of $a_{q-1}^{1} \cup b_{i_{1}}$ and $a_{0}^{1} \cup b_{i_{1}-1}$ intersects $a_{j}^{2}$ at 4 elements, and the other at less than 3 points; (3) exactly one of $a_{q-1}^{1} \cup b_{i_{1}}$ and $a_{0}^{1} \cup b_{i_{1}-1}$ intersects $a_{j}^{2}$ at 3 elements, and the other at less than 3 points. In any case, we have $m_{2} \geq p-1-2 \alpha-8 \beta-2 \times 4 \geq 1$. This means that there exists at least one such index $i_{2} \neq i_{1}$ so that $S_{2}=\left[\ldots, b_{i_{1}-1}, T_{1}, b_{i_{1}}, \ldots, b_{i_{2}-1}, T_{2}, b_{i_{2}}, \ldots\right]$ is a CSCU, where $S_{2}$ is obtained by inserting $T_{2}$ into $S_{1}$ between $b_{i_{2}-1}$ and $b_{i_{2}}$. 
Suppose we have inserted $T_{k}$ into $S_{k-1}$ for $k=1, \ldots, n-1$, and let $S_{k}$ denote the obtained CSCU. We want to insert $T_{k+1}$ into $S_{k}$ between $b_{i_{k+1}-1}$ and $b_{i_{k+1}}$ where $0 \leq i_{k+1} \leq p-1$ and $i_{k+1} \neq i_{l}$ for any $1 \leq l \leq k$. Similarly, we need only care about the unions in $\widehat{S_{0}} \cup\left\{a_{q-1}^{1} \cup b_{i_{1}}, \ldots, a_{q-1}^{k} \cup b_{i_{k}}, a_{0}^{1} \cup b_{i_{1}-1}, \ldots, a_{0}^{k} \cup b_{i_{k}-1}\right\}$. Then we have $m_{k+1} \geq p-k-2 \alpha-8 \beta-8 k$. It is easy to check that $m_{k} \geq 1$ for any $1 \leq k \leq n$. So there exist $n$ distinct indices $0 \leq i_{1}, i_{2}, \ldots, i_{n} \leq p-1$ such that when we insert each $T_{k}$ into $S_{k-1}$ between $b_{i_{k}-1}$ and $b_{i_{k}}$, the obtained sequence is a CSCU-HSQS $(m n+s: s)$ when $1 \leq k \leq n$ or a CSCU-HSQS $(m n+s: m+s)$ when $1 \leq k \leq n-1$.

For a CQS $(X, S, \mathcal{G}, \mathcal{B})$, we may view $S$ as a special group, that is, let $S \in \mathcal{G}$, and we will write CQS $(X, \mathcal{G}, \mathcal{B})$ for convenience. If a block of size $k$ intersects each group in at most one point, we say it is $k$-partite (see [9]). For any design $(X, \mathcal{G}, \mathcal{B})$, GDD, or CQS, let $P$ be a permutation on $X$. For each $G \in \mathcal{G}$, if $P(G)=G$, then the design $(X, \mathcal{G}, P(\mathcal{B}))$ is isomorphic to $(X, \mathcal{G}, \mathcal{B})$. For a point $x \in X$, denote by $\bar{G}_{x}$ the group containing $x$. For a block $B \in \mathcal{B}$, let $P_{B}=\left\{\prod_{x \in B}(x y) \mid y \in\right.$ $\bar{G}_{x}$ and $(x y)$ is a transposition $\}$. Note that each permutation in $P_{B}$ permutes each point of $B$ to a point in the same group and leaves any other point invariant.

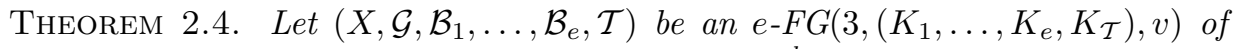
type $g_{1}^{n_{1}} g_{2}^{n_{2}} \ldots g_{r}^{n_{r}}$. Suppose that there exist a $C Q S\left(m^{k_{1}}: s_{1}\right)$ for any $k_{1} \in K_{1}$, a $G D D\left(m^{k_{i}} s_{i}^{1}\right)$ for any $k_{i} \in K_{i}$ with $2 \leq i \leq e$, and $a G D D\left(m^{k}\right)$ for any $k \in K_{\mathcal{T}}$. Then there exists a CQS $\left(\left(m g_{1}\right)^{n_{1}}\left(m g_{2}\right)^{n_{2}} \ldots\left(m g_{r}\right)^{n_{r}}: \sum_{1 \leq i \leq e} s_{i}\right)$. Furthermore, if

(1) the block set of each ingredient design can be arranged into a CSCU, and for any $A \in \mathcal{B}_{1}$, the ingredient $C Q S\left(m^{|A|}: s_{1}\right)$ contains a 4-partite block, and

(2) the master e-fan design has two disjoint blocks $b, b^{\prime} \in \mathcal{T}$ if $e=0$, or $b \in \mathcal{T}$ and $b^{\prime} \in \mathcal{B}_{1}$ if $e \neq 0$,

then there exists a CSCU-CQS $\left(\left(m g_{1}\right)^{n_{1}}\left(m g_{2}\right)^{n_{2}} \ldots\left(m g_{r}\right)^{n_{r}}: \sum_{1 \leq i \leq e} s_{i}\right)$ when $m \geq$ $\max \left\{5, s_{i} \mid 1 \leq i \leq e\right\}$ and $s_{i} \neq 1$ for each $1 \leq i \leq e$.

Proof. Let $I_{l}=\{0,1, \ldots, l-1\}$ for any positive integer $l$ and $I_{0}=\emptyset$. Denote $G_{x}=\{x\} \times I_{m}$ for $x \in X$, and $S_{j}=\left\{\infty_{j}\right\} \times I_{s_{j}}$ for $1 \leq j \leq e$, where $\left\{\infty_{j} \mid 1 \leq j \leq\right.$ $e\} \cap X=\emptyset$. We construct the desired design on $X^{\prime}=\left(X \times I_{m}\right) \cup S$ with the group set $\mathcal{G}^{\prime}=\left\{G \times I_{m} \mid G \in \mathcal{G}\right\}$ and the stem $S=S_{1} \cup S_{2} \cup \cdots \cup S_{e}$. Clearly, $\left(X \times I_{m}\right) \cap S=\emptyset$.

For each block $A \in \mathcal{B}_{1}$, construct a CSCU-CQS $\left(m^{|A|}: s_{1}\right)$ on $X_{A}=\left(A \times I_{m}\right) \cup S_{1}$ having $\left\{G_{x} \mid x \in A\right\}$ as its group set, $S_{1}$ as its stem, and $\mathcal{A}_{A}$ as its block set. Denote $G_{A}=\left\{G_{x} \mid x \in A\right\} \cup\left\{S_{1}\right\}$.

For each block $A \in \mathcal{B}_{j}, 2 \leq j \leq e$, construct a CSCU-GDD $\left(m^{|A|} s_{j}^{1}\right)$ on $X_{A}=$ $\left(A \times I_{m}\right) \cup S_{j}$ having $G_{A}=\left\{G_{x} \mid x \in A\right\} \cup\left\{S_{j}\right\}$ as its group set and $\mathcal{A}_{A}$ as its block set.

For each block $A \in \mathcal{T}$, construct a $\operatorname{CSCU}-\operatorname{GDD}\left(m^{|A|}\right)$ on $X_{A}=A \times I_{m}$ having $G_{A}=\left\{G_{x} \mid x \in A\right\}$ as its group set and $\mathcal{A}_{A}$ as its block set.

Let $\mathcal{B}=\left(\cup_{1 \leq i \leq e} \mathcal{B}_{i}\right) \cup \mathcal{T}$. Then $\cup_{A \in \mathcal{B}} \mathcal{A}_{A}$ is the block set of a CQS $\left(\left(m g_{1}\right)^{n_{1}}\left(m g_{2}\right)^{n_{2}}\right.$ $\left.\ldots\left(m g_{r}\right)^{n_{r}}: \sum_{1 \leq i \leq e} s_{i}\right)$. We try to find a CSCU of $\cup_{A \in \mathcal{B}} \mathcal{A}_{A}$.

First, by our assumption, when $e \neq 0$, we can arrange $\mathcal{B}$ into a sequence $\mathcal{S}^{\prime}=$ $\left[b_{0}, b_{1}, \ldots, b_{p-1}\right]$ where the blocks of $\mathcal{B}_{1}$ are consecutive with $b_{p-2} \in \mathcal{B}_{1}$ being the tail-end, $b_{p-1} \in \mathcal{T}$, and $b_{p-2} \cap b_{p-1}=\emptyset$; when $e=0$, we simply let $b_{p-2} \cap b_{p-1}=\emptyset$. Next, we replace each block $b_{i}$ by a cut CSCU $T_{i}=\left[a_{0}^{i}, a_{1}^{i}, \ldots, a_{q_{i}-1}^{i}\right]$ of $\mathcal{A}_{b_{i}}$, where $a_{0}^{i}$ and $a_{q_{i}-1}^{i}$ are the two ends, and $q_{i}=\left|\mathcal{A}_{b_{i}}\right|, 0 \leq i \leq p-1$. By the hypothesis and the definition of a GDD, without loss of generality, we may assume that $a_{0}^{i}$ intersects each group in $G_{A}$ in at most one point. Now we have the following claim.

ClaIM. There exists a set of permutations $\left\{\sigma_{k} \in P_{a_{0}^{k}} \mid 0 \leq k \leq p-1\right\}$ such that in the cyclic sequence $\mathcal{S}=\left[\sigma_{0}\left(T_{0}\right), \sigma_{1}\left(T_{1}\right), \ldots, \sigma_{p-1}\left(T_{p-1}\right)\right]$, we have $\sigma_{k-1}\left(a_{q_{k-1}-1}^{k-1}\right) \cap$ 
$\sigma_{k}\left(a_{0}^{k}\right)=\emptyset$ and $d\left(\sigma_{l-1}\left(a_{q_{l-1}-1}^{l-1}\right) \cup \sigma_{l}\left(a_{0}^{l}\right), \sigma_{k-1}\left(a_{q_{k-1}-1}^{k-1}\right) \cup \sigma_{k}\left(a_{0}^{k}\right)\right) \geq 4$ for any $0 \leq$ $k, l \leq p-1$, and $|k-l| \geq 2$.

We use a recursive method to prove this claim. Denote $\Gamma_{0}=\left\{\sigma \in P_{a_{0}^{0}} \mid a_{q_{p-1}-1}^{p-1} \cap\right.$ $\left.\sigma\left(a_{0}^{0}\right)=\emptyset\right\} \subseteq P_{a_{0}^{0}}$. From the assumptions on $a_{0}^{i}$ and $b_{p-1}$, we know that $\mid a_{0}^{0} \cap$ $a_{q_{p-1}-1}^{p-1} \mid \leq 2$. We consider all possible intersections of $a_{0}^{0}$ and $a_{q_{p-1}-1}^{p-1}$. Let $a_{0}^{0}=$ $\left\{\left(x_{1}, l_{1}\right),\left(x_{2}, l_{2}\right),\left(x_{3}, l_{3}\right),\left(x_{4}, l_{4}\right)\right\}$ and $a_{q_{p-1}-1}^{p-1}=\left\{\left(y_{1}, l_{1}^{\prime}\right),\left(y_{2}, l_{2}^{\prime}\right),\left(y_{3}, l_{3}^{\prime}\right),\left(y_{4}, l_{4}^{\prime}\right)\right\}$. We first consider the case that $x_{i} \neq \infty_{j}$ for any $1 \leq i \leq 4$ and $1 \leq j \leq e$. If $\left|\left\{x_{1}, x_{2}, x_{3}, x_{4}\right\} \cap\left\{y_{1}, y_{2}, y_{3}, y_{4}\right\}\right|=0$, then $\left|\Gamma_{0}\right|=m^{4}$; if $\mid\left\{x_{1}, x_{2}, x_{3}, x_{4}\right\} \cap\left\{y_{1}, y_{2}, y_{3}\right.$, $\left.y_{4}\right\} \mid=1$, then $\left|\Gamma_{0}\right|=(m-1) m^{3}$; if $\left|\left\{x_{1}, x_{2}, x_{3}, x_{4}\right\} \cap\left\{y_{1}, y_{2}, y_{3}, y_{4}\right\}\right|=2$, then $\left|\Gamma_{0}\right|=(m-1)^{2} m^{2}$. Next we consider the case that $x_{i}=\infty_{j}$ for a unique $1 \leq i \leq 4$ and a unique $1 \leq j \leq e$, which implies that $s_{j} \geq 2$. If $\left|\left\{x_{1}, x_{2}, x_{3}, x_{4}\right\} \cap\left\{y_{1}, y_{2}, y_{3}, y_{4}\right\}\right|=0$, then $\left|\Gamma_{0}\right|=s_{j} m^{3}$; if $\left|\left\{x_{1}, x_{2}, x_{3}, x_{4}\right\} \cap\left\{y_{1}, y_{2}, y_{3}, y_{4}\right\}\right|=1$, then $\left|\Gamma_{0}\right|=s_{j}(m-1) m^{2}$; if $\left|\left\{x_{1}, x_{2}, x_{3}, x_{4}\right\} \cap\left\{y_{1}, y_{2}, y_{3}, y_{4}\right\}\right|=2$, then $\left|\Gamma_{0}\right|=s_{j}(m-1)^{2} m$. So we know that $\left|\Gamma_{0}\right| \geq \min \left\{m^{4},(m-1) m^{3},(m-1)^{2} m^{2}, s_{j} m^{3}, s_{j}(m-1) m^{2}, s_{j}(m-1)^{2} m \mid s_{j} \geq 2\right\} \geq 1$. Choose $\sigma_{0} \in \Gamma_{0}$ and let $\mathcal{S}_{0}=\left\langle\sigma_{0}\left(T_{0}\right)\right\rangle$ be a noncyclic sequence of $\sigma_{0}\left(T_{0}\right)$, that is, $\left\langle\sigma_{0}\left(T_{0}\right)\right\rangle$ is exactly the same as $\left[\sigma_{0}\left(T_{o}\right)\right]$ except that $\sigma\left(a_{0}^{0}\right)$ is not considered as a successor of $\sigma\left(a_{q_{0}-1}^{0}\right)$.

Similarly, we denote $\Gamma_{1}=\left\{\sigma \in P_{a_{0}^{1}} \mid \sigma_{0}\left(a_{q_{0}-1}^{0}\right) \cap \sigma\left(a_{0}^{1}\right)=\emptyset\right\} \subseteq P_{a_{0}^{1}}$. Again, we consider all possible intersections of $a_{0}^{1}$ and $\sigma\left(a_{q_{0}-1}^{0}\right)$. Let $a_{0}^{1}=\left\{\left(x_{1}, l_{1}\right),\left(x_{2}, l_{2}\right),\left(x_{3}, l_{3}\right)\right.$, $\left.\left(x_{4}, l_{4}\right)\right\}$ and $\sigma_{0}\left(a_{q_{0}-1}^{0}\right)=\left\{\left(y_{1}, l_{1}^{\prime}\right),\left(y_{2}, l_{2}^{\prime}\right),\left(y_{3}, l_{3}^{\prime}\right),\left(y_{4}, l_{4}^{\prime}\right)\right\}$. If $y_{i} \neq \infty_{j}$ for any $1 \leq$ $i \leq 4$ and $1 \leq j \leq e$, and all $y_{i}$ are distinct, then $\left|\Gamma_{1}\right|=m^{4}$ or $(m-1) m^{3}$ or $(m-1)^{2} m^{2}$ or $s_{j} m^{3}$ or $s_{j}(m-1) m^{2}$ or $s_{j}(m-1)^{2} m$, with $s_{j} \geq 2$ and $1 \leq j \leq e$, depending on whether $\left|\left\{x_{1}, x_{2}, x_{3}, x_{4}\right\} \cap\left\{y_{1}, y_{2}, y_{3}, y_{4}\right\}\right|$ is equal to 0 or 1 or 2 , and whether $\left|\left\{x_{1}, x_{2}, x_{3}, x_{4}\right\} \cap\left\{\infty_{j} \mid 1 \leq j \leq e\right\}\right|$ is equal to 0 or 1 . If $y_{i} \neq \infty_{j}$ for any $1 \leq i \leq 4$ and $1 \leq j \leq e$, and exactly two of $y_{i}$ are equal, then $\left|\Gamma_{1}\right|=m^{4}$ or $(m-1) m^{3}$ or $(m-2) m^{3}$ or $(m-1)^{2} m^{2}$ or $(m-2)(m-1) m^{2}$ or $s_{j} m^{3}$ or $s_{j}(m-1) m^{2}$ or $s_{j}(m-2) m^{2}$ or $s_{j}(m-1)^{2} m$ or $s_{j}(m-2)(m-1) m$, with $s_{j} \geq 2$ and $1 \leq j \leq e$, depending on whether $\left|\left\{x_{1}, x_{2}, x_{3}, x_{4}\right\} \cap\left\{y_{1}, y_{2}, y_{3}, y_{4}\right\}\right|$ is equal to 0 or 1 or 2 , and whether $\left|\left\{x_{1}, x_{2}, x_{3}, x_{4}\right\} \cap\left\{\infty_{j} \mid 1 \leq j \leq e\right\}\right|$ is equal to 0 or 1 . If $y_{i} \neq \infty_{j}$ for any $1 \leq i \leq 4$ and $1 \leq j \leq e$, and $y_{i_{1}}=y_{i_{2}}$ and $y_{i_{3}}=y_{i_{4}}$, but these two values are not the same, then $\left|\Gamma_{1}\right|=m^{4}$ or $(m-2) m^{3}$ or $(m-2)^{2} m^{2}$ or $s_{j} m^{3}$ or $s_{j}(m-2) m^{2}$ or $s_{j}(m-2)^{2} m$, with $s_{j} \geq 2$ and $1 \leq j \leq e$, depending on whether $\left|\left\{x_{1}, x_{2}, x_{3}, x_{4}\right\} \cap\left\{y_{1}, y_{2}, y_{3}, y_{4}\right\}\right|$ is equal to 0 or 1 or 2 , and whether $\left|\left\{x_{1}, x_{2}, x_{3}, x_{4}\right\} \cap\left\{\infty_{j} \mid 1 \leq j \leq e\right\}\right|$ is equal to 0 or 1 . If $y_{i}=\infty_{j}$ for a unique $1 \leq i \leq 4$ and a unique $1 \leq j \leq e$, then all $y_{i}$ should be distinct, and $\left|\Gamma_{1}\right|=m^{4}$ or $(m-1) m^{3}$ or $(m-1)^{2} m^{2}$ or $s_{i} m^{3}$ or $s_{i}(m-1) m^{2}$ or $\left(s_{j}-1\right) m^{3}$ or $s_{i}(m-1)^{2} m$ or $\left(s_{j}-1\right)(m-1) m^{2}$, with $s_{i} \geq 2, s_{j} \geq 2$, and $1 \leq i \neq j \leq e$, depending on whether $\left|\left\{x_{1}, x_{2}, x_{3}, x_{4}\right\} \cap\left\{y_{1}, y_{2}, y_{3}, y_{4}\right\}\right|$ is equal to 0 or 1 or 2 , and whether $\left|\left\{x_{1}, x_{2}, x_{3}, x_{4}\right\} \cap\left\{\infty_{j} \mid 1 \leq j \leq e\right\}\right|$ is equal to 0 or 1 . In any case, we know that $\left|\Gamma_{1}\right| \geq 1$. Let $\mathcal{S}_{1}=\left\langle\sigma_{0}\left(T_{0}\right), \sigma_{1}\left(T_{1}\right)\right\rangle$, where $\sigma_{1} \in \Gamma_{1}$.

Suppose that there exists a set of permutations $\left\{\sigma_{k} \in P_{a_{0}^{k}} \mid 0 \leq k \leq i-1<p-2\right\}$ such that $\sigma_{k-1}\left(a_{q_{k-1}-1}^{k-1}\right) \cap \sigma_{k}\left(a_{0}^{k}\right)=\emptyset$ and $d\left(\sigma_{l-1}\left(a_{q_{l-1}-1}^{l-1}\right) \cup \sigma_{l}\left(a_{0}^{l}\right), \sigma_{k-1}\left(a_{q_{k-1}-1}^{k-1}\right) \cup\right.$ $\left.\sigma_{k}\left(a_{0}^{k}\right)\right) \geq 4$ for any $0 \leq k, l \leq i-1$, and $|k-l| \geq 2$. Let $\mathcal{S}_{i-1}=\left\langle\sigma_{0}\left(T_{0}\right), \sigma_{1}\left(T_{1}\right), \ldots\right.$, $\left.\sigma_{i-1}\left(T_{i-1}\right)\right\rangle$.

For $k=i$, we try to find a permutation $\sigma_{i} \in P_{a_{0}^{i}}$ such that $\sigma_{i-1}\left(a_{q_{i-1}-1}^{i-1}\right) \cap \sigma_{i}\left(a_{0}^{i}\right)=$ $\emptyset$ and $d\left(\sigma_{i-1}\left(a_{q_{i-1}-1}^{i-1}\right) \cup \sigma_{i}\left(a_{0}^{i}\right), \sigma_{l-1}\left(a_{q_{l-1}-1}^{l-1}\right) \cup \sigma_{l}\left(a_{0}^{l}\right)\right) \geq 4$ for any $0 \leq l<i-1$.

Let $a_{0}^{i}=\left\{\left(x_{1}, l_{1}\right),\left(x_{2}, l_{2}\right),\left(x_{3}, l_{3}\right),\left(x_{4}, l_{4}\right)\right\}$ and $\sigma_{i-1}\left(a_{q_{i-1}-1}^{i-1}\right)=\left\{\left(y_{1}, l_{1}^{\prime}\right),\left(y_{2}, l_{2}^{\prime}\right)\right.$, $\left.\left(y_{3}, l_{3}^{\prime}\right),\left(y_{4}, l_{4}^{\prime}\right)\right\}$. Denote $\Gamma_{i}=\left\{\sigma \in P_{a_{0}^{i}} \mid \sigma_{i-1}\left(a_{q_{i-1}-1}^{i-1}\right) \cap \sigma\left(a_{0}^{i}\right)=\emptyset\right\} \subseteq P_{a_{0}^{i}}$. We first 
divide the problem into two possible cases.

(a) Suppose that $\left\{x_{1}, x_{2}, x_{3}, x_{4}\right\} \cap\left\{y_{1}, y_{2}, y_{3}, y_{4}\right\}=\left\{\infty_{j}\right\}$ for some $j, 1 \leq j \leq e$. Then $s_{j} \geq 2$. For convenience, let $x_{4}=y_{4}=\infty_{j}$. Then $b_{i}, b_{i-1} \in \mathcal{B}_{j}$ and $\left|b_{i} \cap b_{i-1}\right| \leq$ 1. In a way similar to the above analysis, we can prove that $\left|\Gamma_{i}\right| \geq\left(s_{j}-1\right)(m-1) m^{2} \geq$ 1. Now we choose $\sigma_{i, 0} \in \Gamma_{i}$, which satisfies that $\sigma_{i-1}\left(a_{q_{i-1}-1}^{i-1}\right) \cap \sigma_{i, 0}\left(a_{0}^{i}\right)=\emptyset$. If there exists an index $l, 0 \leq l<i-1$, such that $d\left(\sigma_{i-1}\left(a_{q_{i-1}-1}^{i-1}\right) \cup \sigma_{i, 0}\left(a_{0}^{i}\right), \sigma_{l-1}\left(a_{q_{l-1}-1}^{l-1}\right) \cup\right.$ $\left.\sigma_{l}\left(a_{0}^{l}\right)\right)<4$, then exactly one of the two blocks $\left\{b_{l-1}, b_{l}\right\}$ belongs to $\mathcal{B}_{j}$. The reason is explained below. If $b_{l-1}, b_{l} \in \mathcal{B}_{j}$, then since $b_{i-1}, b_{i} \in \mathcal{B}_{j}$, we know that $\left|\left(b_{i} \cup b_{i-1}\right) \cap\left(b_{l-1} \cup b_{l}\right)\right| \leq\left|b_{i} \cap b_{l-1}\right|+\left|b_{i-1} \cap b_{l-1}\right|+\left|b_{i} \cap b_{l}\right|+\left|b_{i-1} \cap b_{l}\right| \leq 4$, and hence $\left|\left(\sigma_{i-1}\left(a_{q_{i-1}-1}^{i-1}\right) \cup \sigma_{i, 0}\left(a_{0}^{i}\right)\right) \cap\left(\sigma_{l-1}\left(a_{q_{l-1}-1}^{l-1}\right) \cup \sigma_{l}\left(a_{0}^{l}\right)\right)\right| \leq 6$, which is impossible, for in this case we would have $d\left(\sigma_{i-1}\left(a_{q_{i-1}-1}^{i-1}\right) \cup \sigma_{i, 0}\left(a_{0}^{i}\right), \sigma_{l-1}\left(a_{q_{l-1}-1}^{l-1}\right) \cup \sigma_{l}\left(a_{0}^{l}\right)\right) \geq 4$. On the other hand, if $b_{l-1}, b_{l} \notin \mathcal{B}_{j}$, then $\left(\infty_{j}, l_{4}^{\prime \prime}\right) \notin \sigma_{l-1}\left(a_{q_{l-1}-1}^{l-1}\right) \cup \sigma_{l}\left(a_{0}^{l}\right)$ for any $l_{4}^{\prime \prime} \in I_{s_{j}}$, so $\left|\left(\sigma_{i-1}\left(a_{q_{i-1}-1}^{i-1}\right) \cup \sigma_{i, 0}\left(a_{0}^{i}\right)\right) \cap\left(\sigma_{l-1}\left(a_{q_{l-1}-1}^{l-1}\right) \cup \sigma_{l}\left(a_{0}^{l}\right)\right)\right| \leq 6$, which is again impossible. Then there are two cases to be considered: $b_{l-1} \in \mathcal{B}_{j}, b_{l} \notin \mathcal{B}_{j}$ and $b_{l} \in \mathcal{B}_{j}$, $b_{l-1} \notin \mathcal{B}_{j}$. We first assume that $b_{l-1} \in \mathcal{B}_{j}$ and $b_{l} \notin \mathcal{B}_{j}$. Then clearly $\sigma_{l}\left(a_{0}^{l}\right) \cap S_{j}=\emptyset$. Since we have supposed that $d\left(\sigma_{i-1}\left(a_{q_{i-1}-1}^{i-1}\right) \cup \sigma_{i, 0}\left(a_{0}^{i}\right), \sigma_{l-1}\left(a_{q_{l-1}-1}^{l-1}\right) \cup \sigma_{l}\left(a_{0}^{l}\right)\right)<$ 4, i.e., $\left|\left(\sigma_{i-1}\left(a_{q_{i-1}-1}^{i-1}\right) \cup \sigma_{i, 0}\left(a_{0}^{i}\right)\right) \cap\left(\sigma_{l-1}\left(a_{q_{l-1}-1}^{l-1}\right) \cup \sigma_{l}\left(a_{0}^{l}\right)\right)\right| \geq 7$, we should have that one of $\left|\sigma_{l-1}\left(a_{q_{l-1}-1}^{l-1}\right) \cap\left(\sigma_{i-1}\left(a_{q_{i-1}-1}^{i-1}\right) \cup \sigma_{i, 0}\left(a_{0}^{i}\right)\right)\right|$ and $\mid \sigma_{l}\left(a_{0}^{l}\right) \cap\left(\sigma_{i-1}\left(a_{q_{i-1}-1}^{i-1}\right) \cup\right.$ $\left.\sigma_{i, 0}\left(a_{0}^{i}\right)\right) \mid$ equals 4 and the other at least 3 . Since $b_{l-1} \in \mathcal{B}_{j}$, then $\left|b_{l-1} \cap b_{i}\right| \leq 1$ and $\left|b_{l-1} \cap b_{i-1}\right| \leq 1$, i.e., $\left|\left(b_{l-1} \cup\left\{\infty_{j}\right\}\right) \cap\left(b_{i} \cup b_{i-1} \cup\left\{\infty_{j}\right\}\right)\right| \leq 3$, which implies that $\left|\sigma_{l-1}\left(a_{q_{l-1}-1}^{l-1}\right) \cap\left(\sigma_{i-1}\left(a_{q_{i-1}-1}^{i-1}\right) \cup \sigma_{i, 0}\left(a_{0}^{i}\right)\right)\right| \leq 3$. Therefore, it is necessary that $\left|\sigma_{l-1}\left(a_{q_{l-1}-1}^{l-1}\right) \cap\left(\sigma_{i-1}\left(a_{q_{i-1}-1}^{i-1}\right) \cup \sigma_{i, 0}\left(a_{0}^{i}\right)\right)\right|=3$ and $\mid \sigma_{l}\left(a_{0}^{l}\right) \cap\left(\sigma_{i-1}\left(a_{q_{i-1}-1}^{i-1}\right) \cup\right.$ $\left.\sigma_{i, 0}\left(a_{0}^{i}\right)\right) \mid=4$. Then we can let $\sigma_{l-1}\left(a_{q_{l-1}-1}^{l-1}\right)=\left\{\left(x_{1}, \sigma_{i, 0}\left(l_{1}\right)\right),\left(y_{1}, l_{1}^{\prime}\right),\left(\infty_{j}, \diamond\right),(\star, *)\right\}$ and $\sigma_{l}\left(a_{0}^{l}\right)=\left\{\left(x_{2}, \sigma_{i, 0}\left(l_{2}\right)\right),\left(x_{3}, \sigma_{i, 0}\left(l_{3}\right)\right),\left(y_{2}, l_{2}^{\prime}\right),\left(y_{3}, l_{3}^{\prime}\right)\right\}$, where $\diamond \in\left\{\sigma_{i, 0}\left(l_{4}\right), l_{4}^{\prime}\right\}$. If $\diamond=\sigma_{i, 0}\left(l_{4}\right)$, no permutation $\sigma \in \Gamma_{i}$ satisfies that $\mid\left(\sigma_{i-1}\left(a_{q_{i-1}-1}^{i-1}\right) \cup \sigma\left(a_{0}^{i}\right)\right) \cap$ $\left(\sigma_{l-1}\left(a_{q_{l-1}-1}^{l-1}\right) \cup \sigma_{l}\left(a_{0}^{l}\right)\right) \mid \geq 7$ except $\sigma=\sigma_{i, 0}$. If $\diamond=l_{4}^{\prime}$, then all the permutations $\sigma_{i, 1} \in \Gamma_{i}$ which change $\left(\infty_{j}, \sigma_{i, 0}\left(l_{4}\right)\right)$ to every element in $\left\{\infty_{j}\right\} \times\left(Z_{s_{j}} \backslash\left\{l_{4}^{\prime}\right\}\right)$ and fix the other three points in $\sigma_{i, 0}\left(a_{0}^{i}\right)$ satisfy that $\mid\left(\sigma_{i-1}\left(a_{q_{i-1}-1}^{i-1}\right) \cup \sigma_{i, 1}\left(\sigma_{i, 0}\left(a_{0}^{i}\right)\right)\right) \cap$ $\left(\sigma_{l-1}\left(a_{q_{l-1}-1}^{l-1}\right) \cup \sigma_{l}\left(a_{0}^{l}\right)\right) \mid \geq 7$. So for such a pair $\left(b_{l-1}, b_{l}\right)$, there are at most $\left(s_{j}-1\right)$ $\sigma \in \Gamma_{i}$ such that $d\left(\sigma_{i-1}\left(a_{q_{i-1}-1}^{i-1}\right) \cup \sigma\left(a_{0}^{i}\right), \sigma_{l-1}\left(a_{q_{l-1}-1}^{l-1}\right) \cup \sigma_{l}\left(a_{0}^{l}\right)\right)<4$. Now we compute the number of such pairs $\left(b_{l-1}, b_{l}\right)$ or, equivalently, the number of such $b_{l}$. There are $\left(\begin{array}{l}6 \\ 3\end{array}\right)-2=18$ triples in $\left\{x_{1}, x_{2}, x_{3}, y_{1}, y_{2}, y_{3}\right\}$ excluding the two triples $\left\{x_{1}, x_{2}, x_{3}\right\}$ and $\left\{y_{1}, y_{2}, y_{3}\right\}$. Since each triple occurs in exactly one block of $\mathcal{B}$, each block of $\mathcal{B}$ contains exactly 4 triples, $\left|b_{l} \cap\left\{x_{1}, x_{2}, x_{3}\right\}\right|=2$, and $\left|b_{l} \cap\left\{y_{1}, y_{2}, y_{3}\right\}\right|=2$, we know that there are at most $\lfloor 18 / 4\rfloor=4$ such $b_{l}$. From the assumption, we have $\left|\Gamma_{i}\right| \geq(m-1) m^{2}\left(s_{j}-1\right)>4\left(s_{j}-1\right)$, which implies that there exists at least one permutation $\sigma_{i} \in \Gamma_{i}$ such that $\sigma_{i-1}\left(a_{q_{i-1}-1}^{i-1}\right) \cap \sigma_{i}\left(a_{0}^{i}\right)=\emptyset$ and $d\left(\sigma_{i-1}\left(a_{q_{i-1}-1}^{i-1}\right) \cup\right.$ $\left.\sigma_{i}\left(a_{0}^{i}\right), \sigma_{l-1}\left(a_{q_{l-1}-1}^{l-1}\right) \cup \sigma_{l}\left(a_{0}^{l}\right)\right) \geq 4$ for any $0 \leq l<i-1$. For the case that $b_{l} \in \mathcal{B}_{j}$ and $b_{l-1} \notin \mathcal{B}_{j}$, we can also prove, in the same fashion as above, that the same assertion holds.

(b) Suppose that $\left\{x_{1}, x_{2}, x_{3}, x_{4}\right\} \cap\left\{y_{1}, y_{2}, y_{3}, y_{4}\right\} \neq\left\{\infty_{j}\right\}$ for any $j, 1 \leq j \leq e$. We further divide this case into two possible subcases.

(b.1) $y_{1}=y_{2}=x_{1}$ and $y_{3}=x_{2}$, i.e., $\sigma_{i-1}\left(a_{q_{i-1}-1}^{i-1}\right)=\left\{\left(x_{1}, l_{1}^{\prime}\right),\left(x_{1}, l_{2}^{\prime}\right),\left(x_{2}, l_{3}^{\prime}\right),\left(y_{4}\right.\right.$, $\left.\left.l_{4}^{\prime}\right)\right\}$. If $y_{4}=x_{2}$, then $\left|\Gamma_{i}\right| \geq \min \left\{(m-2)^{2} m^{2},(m-2)^{2} m s_{j} \mid s_{j} \geq 2\right\} \geq 1$. If $y_{4} \neq x_{2}$, then $\left|\Gamma_{i}\right| \geq \min \left\{(m-2)(m-1) m^{2},(m-2)(m-1) m s_{j} \mid s_{j} \geq 2\right\} \geq 1$. In any case, we know that $\left|\Gamma_{i}\right| \geq 1$. Assume that $\sigma_{i-1}\left(a_{q_{i-1}-1}^{i-1}\right) \cap \sigma_{i, 0}\left(a_{0}^{i}\right)=\emptyset$. We now 
prove that $d\left(\sigma_{i-1}\left(a_{q_{i-1}-1}^{i-1}\right) \cup \sigma_{i, 0}\left(a_{0}^{i}\right), \sigma_{l-1}\left(a_{q_{l-1}-1}^{l-1}\right) \cup \sigma_{l}\left(a_{0}^{l}\right)\right) \geq 4$ for $0 \leq l \leq i-1$. If $\left\{x_{1}, x_{2}\right\} \subset b_{l}$, then $\left|b_{l} \cap\left(b_{i-1} \cup b_{i}\right)\right|=2$. Since $a_{0}^{l}$ is 4-partite, we know that $\left|\left(\sigma_{i-1}\left(a_{q_{i-1}-1}^{i-1}\right) \cup \sigma_{i, 0}\left(a_{0}^{i}\right)\right) \cap \sigma_{l}\left(a_{0}^{l}\right)\right| \leq 2$, which makes $d\left(\sigma_{i-1}\left(a_{q_{i-1}-1}^{i-1}\right) \cup \sigma_{i, 0}\left(a_{0}^{i}\right), \sigma_{l-1}\right.$ $\left.\left(a_{q_{l-1}-1}^{l-1}\right) \cup \sigma_{l}\left(a_{0}^{l}\right)\right) \geq 4$. If $\left\{x_{1}, x_{2}\right\} \subset b_{l-1}$, then since $y_{1}=y_{2}=x_{1}$, we know that $b_{i-1} \in \mathcal{B}_{1}$ and thus $b_{l-1} \notin \mathcal{B}_{1}$, implying that $a_{q_{l-1}-1}^{l-1}$ is a block in some ingredient GDD and therefore is 4-partite. So $\left|\left(\sigma_{i-1}\left(a_{q_{i-1}-1}^{i-1}\right) \cup \sigma_{i, 0}\left(a_{0}^{i}\right)\right) \cap \sigma_{l-1}\left(a_{q_{l-1}-1}^{l-1}\right)\right| \leq 2$, which ensures that $d\left(\sigma_{i-1}\left(a_{q_{i-1}-1}^{i-1}\right) \cup \sigma_{i, 0}\left(a_{0}^{i}\right), \sigma_{l-1}\left(a_{q_{l-1}-1}^{l-1}\right) \cup \sigma_{l}\left(a_{0}^{l}\right)\right) \geq 4$. We still need to consider the case when $\left\{x_{1}, x_{2}\right\} \not \subset b_{l-1}$ and $\left\{x_{1}, x_{2}\right\} \not \subset b_{l}$. If $y_{4}=x_{2}$, then $\left|\left(\sigma_{i-1}\left(a_{q_{i-1}-1}^{i-1}\right) \cup \sigma_{i, 0}\left(a_{0}^{i}\right)\right) \cap \sigma_{l}\left(a_{0}^{l}\right)\right| \leq 2$, which makes again $d\left(\sigma_{i-1}\left(a_{q_{i-1}-1}^{i-1}\right) \cup\right.$ $\left.\sigma_{i, 0}\left(a_{0}^{i}\right), \sigma_{l-1}\left(a_{q_{l-1}-1}^{l-1}\right) \cup \sigma_{l}\left(a_{0}^{l}\right)\right) \geq 4$. If $y_{4} \neq x_{2}$, then $\mid\left(\sigma_{i-1}\left(a_{q_{i-1}-1}^{i-1}\right) \cup \sigma_{i, 0}\left(a_{0}^{i}\right)\right) \cap$ $\sigma_{l}\left(a_{0}^{l}\right) \mid \leq 3$ and $\left|\left(\sigma_{i-1}\left(a_{q_{i-1}-1}^{i-1}\right) \cup \sigma_{i, 0}\left(a_{0}^{i}\right)\right) \cap \sigma_{l-1}\left(a_{q_{l-1}-1}^{l-1}\right)\right| \leq 3$, which also ensures that $d\left(\sigma_{i-1}\left(a_{q_{i-1}-1}^{i-1}\right) \cup \sigma_{i, 0}\left(a_{0}^{i}\right), \sigma_{l-1}\left(a_{q_{l-1}-1}^{l-1}\right) \cup \sigma_{l}\left(a_{0}^{l}\right)\right) \geq 4$.

(b.2) All cases except (b.1), that is, $\sigma_{i-1}\left(a_{q_{i-1}-1}^{i-1}\right) \in\left\{\left\{\left(x_{1}, l_{1}^{\prime}\right),\left(x_{1}, l_{2}^{\prime}\right),\left(y_{3}, l_{3}^{\prime}\right),\left(y_{4}\right.\right.\right.$, $\left.\left.l_{4}^{\prime}\right)\right\},\left\{\left(x_{1}, l_{1}^{\prime}\right),\left(x_{2}, l_{2}^{\prime}\right),\left(y_{3}, l_{3}^{\prime}\right),\left(y_{4}, l_{4}^{\prime}\right)\right\},\left\{\left(x_{1}, l_{1}^{\prime}\right),\left(y_{2}, l_{2}^{\prime}\right),\left(y_{3}, l_{3}^{\prime}\right),\left(y_{4}, l_{4}^{\prime}\right)\right\},\left\{\left(y_{1}, l_{1}^{\prime}\right),\left(y_{2}\right.\right.$, $\left.\left.\left.l_{2}^{\prime}\right),\left(y_{3}, l_{3}^{\prime}\right),\left(y_{4}, l_{4}^{\prime}\right)\right\}\right\}$, where $y_{i} \neq x_{j}$ for any $i$ anf $j$. A tedious calculation shows that $\left|\Gamma_{i}\right| \geq \min \left\{(m-2) m^{3},(m-2) m^{2} s_{j},(m-1)^{2} m^{2},(m-1)^{2} m s_{j},(m-1) m^{3},(m-\right.$ 1) $\left.m^{2} s_{j}, m^{4}, m^{3} s_{j} \mid s_{j} \geq 2\right\} \geq 2(m-2) m^{2} \geq 1$. Choose $\sigma_{i, 0} \in \Gamma_{i}$. Then $\sigma_{i-1}\left(a_{q_{i-1}-1}^{i-1}\right) \cap$ $\sigma_{i, 0}\left(a_{0}^{i}\right)=\emptyset$. If there exists an index $l, 0 \leq l<i-1$, such that $d\left(\sigma_{i-1}\left(a_{q_{i-1}-1}^{i-1}\right) \cup\right.$ $\left.\sigma_{i, 0}\left(a_{0}^{i}\right), \sigma_{l-1}\left(a_{q_{l-1}-1}^{l-1}\right) \cup \sigma_{l}\left(a_{0}^{l}\right)\right)<4$, then $\mid\left(\sigma_{i-1}\left(a_{q_{i-1}-1}^{i-1}\right) \cup \sigma_{i, 0}\left(a_{0}^{i}\right)\right) \cap\left(\sigma_{l-1}\left(a_{q_{l-1}-1}^{l-1}\right) \cup\right.$ $\left.\sigma_{l}\left(a_{0}^{l}\right)\right) \mid \geq 7$. Let $R=\left\{r \subset X^{\prime} \mid r \subset \sigma_{i-1}\left(a_{q_{i-1}-1}^{i-1}\right) \cup \sigma_{i, 0}\left(a_{0}^{i}\right), r \not \subset \sigma_{i-1}\left(a_{q_{i-1}-1}^{i-1}\right), r \not \subset\right.$ $\sigma_{i, 0}\left(a_{0}^{i}\right)$, and $\left.|r|=3\right\}$; then $|R| \leq\left(\begin{array}{l}4 \\ 2\end{array}\right) \times\left(\begin{array}{l}4 \\ 1\end{array}\right) \times 2=48$. Suppose that there are $t_{1} l$ 's such that $\left|\left(\sigma_{i-1}\left(a_{q_{i-1}-1}^{i-1}\right) \cup \sigma_{i, 0}\left(a_{0}^{i}\right)\right) \cap\left(\sigma_{l-1}\left(a_{q_{l-1}-1}^{l-1}\right) \cup \sigma_{l}\left(a_{0}^{l}\right)\right)\right|=8$ and $t_{2} l$ 's such that $\left|\left(\sigma_{i-1}\left(a_{q_{i-1}-1}^{i-1}\right) \cup \sigma_{i, 0}\left(a_{0}^{i}\right)\right) \cap\left(\sigma_{l-1}\left(a_{q_{l-1}-1}^{l-1}\right) \cup \sigma_{l}\left(a_{0}^{l}\right)\right)\right|=7$. For the former case, each block in $\left\{\sigma_{l-1}\left(a_{q_{l-1}-1}^{l-1}\right), \sigma_{l}\left(a_{0}^{l}\right)\right\}$ contains four triples from $R$. Even if there is one point in one of these two blocks with its second component changed, we still have $\left|\left(\sigma_{i-1}\left(a_{q_{i-1}-1}^{i-1}\right) \cup \sigma_{i, 0}\left(a_{0}^{i}\right)\right) \cap\left(\sigma_{l-1}\left(a_{q_{l-1}-1}^{l-1}\right) \cup \sigma_{l}\left(a_{0}^{l}\right)\right)\right| \geq 7$, that is, $d\left(\sigma_{i-1}\left(a_{q_{i-1}-1}^{i-1}\right) \cup\right.$ $\left.\sigma_{i, 0}\left(a_{0}^{i}\right)\right),\left(\sigma_{l-1}\left(a_{q_{l-1}-1}^{l-1}\right) \cup \sigma_{l}\left(a_{0}^{l}\right)\right)<4$. So there are at $\operatorname{most} \max \{4(m-1)+1,3(m-1)+$ $\left.\left(s_{j}-1\right)+1 \mid s_{j} \geq 2\right\}=4 m-3 \sigma \in \Gamma_{i}$ such that $d\left(\sigma_{i-1}\left(a_{q_{i-1}-1}^{i-1}\right) \cup \sigma\left(a_{0}^{i}\right), \sigma_{l-1}\left(a_{q_{l-1}-1}^{l-1}\right) \cup\right.$ $\left.\sigma_{l}\left(a_{0}^{l}\right)\right)<4$. For the latter case, one block in $\left\{\sigma_{l-1}\left(a_{q_{l-1}-1}^{l-1}\right), \sigma_{l}\left(a_{0}^{l}\right)\right\}$ contains four triples and the other one contains only one triple from $R$. Even if the second component of the uncommon point is changed in the block which contains only one triple from $R$, we still have $\left|\left(\sigma_{i-1}\left(a_{q_{i-1}-1}^{i-1}\right) \cup \sigma_{i, 0}\left(a_{0}^{i}\right)\right) \cap\left(\sigma_{l-1}\left(a_{q_{l-1}-1}^{l-1}\right) \cup \sigma_{l}\left(a_{0}^{l}\right)\right)\right| \geq 7$, that is, $d\left(\sigma_{i-1}\left(a_{q_{i-1}-1}^{i-1}\right) \cup \sigma_{i, 0}\left(a_{0}^{i}\right)\right),\left(\sigma_{l-1}\left(a_{q_{l-1}-1}^{l-1}\right) \cup \sigma_{l}\left(a_{0}^{l}\right)\right)<4$. So there are at most $\max \left\{m, s_{j} \mid s_{j} \geq 2\right\}=m \sigma \in \Gamma_{i}$ such that $d\left(\sigma_{i-1}\left(a_{q_{i-1}-1}^{i-1}\right) \cup \sigma\left(a_{0}^{i}\right), \sigma_{l-1}\left(a_{q_{l-1}-1}^{l-1}\right) \cup\right.$ $\left.\sigma_{l}\left(a_{0}^{l}\right)\right)<4$. Here, $8 t_{1}+5 t_{2} \leq|R| \leq 48$. Since $t_{1} \leq|R| / 8 \leq 6$, we have $t_{1}(4 m-3)+$ $t_{2} m \leq t_{1}(4 m-3)+\left(48-8 t_{1}\right) m / 5=t_{1}(2.4 m-3)+9.6 m \leq 6(2.4 m-3)+9.6 m=24 m-$ 18 . From the assumption that $m \geq 5$, we have $2(m-2) m^{2}>24 m-18$, which implies that there exists at least one permutation $\sigma_{i} \in \Gamma_{i}$ such that $\sigma_{i-1}\left(a_{q_{i-1}-1}^{i-1}\right) \cap \sigma_{i}\left(a_{0}^{i}\right)=\emptyset$ and $d\left(\sigma_{i-1}\left(a_{q_{i-1}-1}^{i-1}\right) \cup \sigma_{i}\left(a_{0}^{i}\right), \sigma_{l-1}\left(a_{q_{l-1}-1}^{l-1}\right) \cup \sigma_{l}\left(a_{0}^{l}\right)\right) \geq 4$ for any $0 \leq l<i-1$.

Then we set $\mathcal{S}_{i}=\left\langle\mathcal{S}_{i-1}, \sigma_{i}\left(T_{i}\right)\right\rangle$ for $1 \leq i \leq p-2$.

When $k=p-1$, we want to find a permutation $\sigma_{p-1} \in P_{a_{0}^{p-1}}$ such that $\sigma_{p-2}\left(a_{q_{p-2}-1}^{p-2}\right) \cap \sigma_{p-1}\left(a_{0}^{p-1}\right)=\emptyset, \sigma_{p-1}\left(a_{q_{p-1}-1}^{p-1}\right)=a_{q_{p-1}-1}^{p-1}$, and $d\left(\sigma_{p-2}\left(a_{q_{p-2}-1}^{p-2}\right) \cap\right.$ $\left.\sigma_{p-1}\left(a_{0}^{p-1}\right), \sigma_{l-1}\left(a_{q_{l-1}-1}^{l-1}\right) \cup \sigma_{l}\left(a_{0}^{l}\right)\right) \geq 4$ for any $1 \leq l<p-2$. By assumption, 
$b_{p-2} \cap b_{p-1}=\emptyset$. This implies that $\sigma_{p-2}\left(a_{q_{p-2}-1}^{p-2}\right) \cap \sigma_{p-1}\left(a_{0}^{p-1}\right)=\emptyset$ for any $\sigma_{p-1} \in$ $P_{a_{0}^{p-1}}$. Denote $\Gamma_{p-1}=\left\{\sigma \in P_{a_{0}^{p-1}} \mid \sigma\left(a_{q_{p-1}-1}^{p-1}\right)=a_{q_{p-1}-1}^{p-1}\right\}$. Since $b_{p-1} \in \mathcal{T}$ is replaced by the cut CSCU $T_{p-1}=\left[a_{0}^{p-1}, a_{1}^{p-1}, \ldots, a_{q_{p-1}-1}^{p-1}\right]$, then, as we said in section 1 , we have that $a_{0}^{p-1} \cap a_{q_{p-1}-1}^{p-1}=\emptyset$. This, together with $\sigma\left(a_{q_{p-1}-1}^{p-1}\right)=a_{q_{p-1}-1}^{p-1}$, shows that $\left|\Gamma_{p-1}\right| \geq(m-1)^{4} \geq 1$. Similar to the proof in (b.2), we can prove that there are at most $(24 m-18) \sigma \in \Gamma_{i}$ such that $d\left(\sigma_{p-2}\left(a_{q_{p-2}-1}^{p-2}\right) \cap \sigma\left(a_{0}^{p-1}\right), \sigma_{l-1}\left(a_{q_{l-1}-1}^{l-1}\right) \cup \sigma_{l}\left(a_{0}^{l}\right)\right)<4$ for some $l, 1 \leq l<p-2$. From the assumption, we have $(m-1)^{4}>24 m-18$. Thus we have proved the existence of $\sigma_{p-1}$.

Now we have finished the proof of the claim. For convenience, we use $T_{k}$ to denote $\sigma_{k}\left(T_{k}\right)$ for $0 \leq k \leq p-1$. Then $\mathcal{S}=\left[T_{0}, T_{1}, \ldots, T_{p-1}\right]$ satisfies the conditions that $a_{q_{k-1}-1}^{k-1} \cap a_{0}^{k}=\emptyset$ and $d\left(a_{q_{l-1}-1}^{l-1} \cup a_{0}^{l}, a_{q_{k-1}-1}^{k-1} \cup a_{0}^{k}\right) \geq 4$ for any $0 \leq k, l \leq p-1$, and $|k-l| \geq 2$. Next, we will prove $\mathcal{S}$ is actually a CSCU.

To do this, we should check the distance between any two elements of $C=$ $\left(\bigcup_{i=0}^{p-1} \mathcal{A}_{b_{i}}\right) \cup\left(\bigcup_{i=0}^{p-1} \overline{T_{i}}\right) \cup\left(\bigcup_{i=0}^{p-1}\left\{a_{q_{i-1}-1}^{i-1} \cup a_{0}^{i}\right\}\right)$. Elements of $C$ are classified into three types.

Type I: $a \in \mathcal{A}_{b_{i}}$ for some $i, 0 \leq i \leq p-1$. If $b_{i} \in \mathcal{B}_{1}$, we say that $a$ belongs to Type $\mathrm{I}_{\mathrm{CQS}}$; otherwise, $a$ belongs to Type $\mathrm{I}_{\mathrm{GDD}}$.

Type II: $c \in \overline{T_{i}}$ for some $i, 0 \leq i \leq p-1$. If $b_{i} \in \mathcal{B}_{1}$, we say that $c$ belongs to Type $\mathrm{II}_{\mathrm{CQS}}$; otherwise, $c$ belongs to Type $\mathrm{II}_{\mathrm{GDD}}$.

Type III: $c \in \bigcup_{i=0}^{p-1}\left\{a_{q_{i-1}-1}^{i-1} \cup a_{0}^{i}\right\}$.

Since the resultant design is a CQS, we easily know the following:

Case 1: $d\left(a, a^{\prime}\right) \geq 4$ for any two distinct $a, a^{\prime}$ from Type I;

Case 2: $d(a, c) \geq 4$ for any $a$ from Type I and $c$ from Types II, III, respectively. Since each $T_{i}, 0 \leq i \leq p-1$, is a CSCU, we have

Case 3: $d\left(c, c^{\prime}\right) \geq 4$ for any $c, c^{\prime} \in \overline{T_{i}}, 0 \leq i \leq p-1$, from Type II.

For each $a \in \mathcal{A}_{b_{i}}$ from Type $\mathrm{I}_{\mathrm{GDD}},\left|a \cap X_{b_{j}}\right| \leq 2$ when $a \notin \mathcal{A}_{b_{j}}$, so

Case 4: $d\left(c, c^{\prime}\right) \geq 4$ for any $c \in \overline{T_{i}}$ from Type $\mathrm{II}_{\mathrm{GDD}}$ and $c^{\prime} \in \overline{T_{j}}$ from Type II.

For each $a \in \mathcal{A}_{b_{i}}$ from Type $\mathrm{I}_{\mathrm{CQS}}$, we know that $b_{i} \in \mathcal{B}_{1}$ and $\left|a \cap X_{b_{j}}\right| \leq 2$ when $b_{j} \in \mathcal{B}_{1}$ and $a \notin \mathcal{A}_{b_{j}}$, so

Case 5: $d\left(c, c^{\prime}\right) \geq 4$ for any $c, c^{\prime}$ from Type $\mathrm{II}_{\mathrm{CQS}}$.

If $b_{i} \notin \mathcal{B}_{1}$, then by the definition of a GDD and our special arrangement of $\mathcal{B}$ into $\mathcal{S}^{\prime}=\left[b_{0}, b_{1}, \ldots, b_{p-1}\right]$, we know that $\left|a \cap a^{\prime}\right| \leq 1$ for any $a \in \mathcal{A}_{b_{i}}$ and $a^{\prime} \in \mathcal{A}_{b_{i-1}}$, so

Case 6: $d\left(c, a_{q_{i-1}-1}^{i-1} \cup a_{0}^{i}\right) \geq 4$ for any $c \in \overline{T_{i}}$ from Type $\mathrm{II}_{\mathrm{GDD}}$.

If $b_{i} \in \mathcal{B}_{1}$, then $\left|a \cap X_{b_{i}}\right| \leq 2$ for any $a \notin \mathcal{A}_{b_{i}}$, so

Case 7: $d\left(c, a_{q_{i-1}-1}^{i-1} \cup a_{0}^{i}\right) \geq 4$ for any $c \in \overline{T_{i}}$ from Type $\mathrm{II}_{\mathrm{CQS}}$.

Since $a_{0}^{j}$ is 4-partite, we know that $\left|a_{0}^{j} \cap X_{b_{i}}\right| \leq 2$ for any $1 \leq i \neq j \leq p-1$, and then

Case 8: $d\left(c, a_{q_{j-1}-1}^{j-1} \cup a_{0}^{j}\right) \geq 4$ for any $c \in \overline{T_{i}}$ and $1 \leq i \neq j \leq p-1$.

Since $a_{0}^{i} \cap a_{q_{i}-1}^{i}=\emptyset$ and $\left|a_{q_{i-1}-1}^{i-1} \cap a_{q_{i}-1}^{i}\right| \leq 2$, we have

Case 9: $d\left(a_{q_{i-1}-1}^{i-1} \cup a_{0}^{i}, a_{q_{i}-1}^{i} \cup a_{0}^{i+1}\right) \geq 4,0 \leq i \leq p-1$.

From the property of $\mathcal{S}$, we know that

Case 10: $d\left(a_{q_{i-1}-1}^{i-1} \cup a_{0}^{i}, a_{q_{l-1}-1}^{l-1} \cup a_{0}^{l}\right) \geq 4$ for $|i-l| \geq 2$.

Then we have proved that $\mathcal{S}$ is in fact a CSCU.

TheOREM 2.5. In Theorem 2.4, if we change the condition (1) to be

$\left(1^{\prime}\right)$ the block set of each ingredient design can be arranged into a CSCU with two consecutive 4-partite blocks,

then there exists a CSCU-CQS $\left(\left(m g_{1}\right)^{n_{1}}\left(m g_{2}\right)^{n_{2}} \ldots\left(m g_{r}\right)^{n_{r}}: \sum_{1 \leq i \leq e} s_{i}\right)$ when $m \geq$ $\max \left\{4, s_{i} \mid 1 \leq i \leq e\right\}$ and $s_{i} \neq 1$ for each $1 \leq i \leq e$. 
Proof. Since the proof is similar to that of Theorem 2.4, we will look at only those places which are different from Theorem 2.4.

First, without loss of generality, we may assume that both $a_{0}^{i}$ and $a_{q_{i}-1}^{i}$ of $T_{i}=$ $\left[a_{0}^{i}, a_{1}^{i}, \ldots, a_{q_{i}-1}^{i}\right]$ are 4-partite for any $i, 0 \leq i \leq p-1$.

Remember that in the proof of Theorem 2.4, we need $m=5$ only in Case (b.2). So we can omit the proof for all cases except for (b.2). We divide Case (b.2) into two subcases.

(b.2.1) If $x_{i}=\infty_{j}$ for some $i$ and $j, 1 \leq i \leq 4,1 \leq j \leq e$, then $\left|\Gamma_{i}\right| \geq \min \{(m-$ $\left.1)^{2} m s_{j},(m-1) m^{2} s_{j}, m^{3} s_{j} \mid s_{j} \geq 2\right\}=(m-1)^{2} m s_{j} \geq 1$. Assume that $\sigma_{i-1}\left(a_{q_{i-1}-1}^{i-1}\right) \cap$ $\sigma_{i, 0}\left(a_{0}^{i}\right)=\emptyset$. If there exists an index $l, 0 \leq l<i-1$, such that $d\left(\sigma_{i-1}\left(a_{q_{i-1}-1}^{i-1}\right) \cup\right.$ $\left.\sigma_{i, 0}\left(a_{0}^{i}\right), \sigma_{l-1}\left(a_{q_{l-1}-1}^{l-1}\right) \cup \sigma_{l}\left(a_{0}^{l}\right)\right)<4$, then, as we knew already in case (b.2) of Theorem 2.4, $\left|\left(\sigma_{i-1}\left(a_{q_{i-1}-1}^{i-1}\right) \cup \sigma_{i, 0}\left(a_{0}^{i}\right)\right) \cap\left(\sigma_{l-1}\left(a_{q_{l-1}-1}^{l-1}\right) \cup \sigma_{l}\left(a_{0}^{l}\right)\right)\right| \geq 7$. Again, let $R=$ $\left\{r \subset X^{\prime} \mid r \subset \sigma_{i-1}\left(a_{q_{i-1}-1}^{i-1}\right) \cup \sigma_{i, 0}\left(a_{0}^{i}\right), r \not \subset \sigma_{i-1}\left(a_{q_{i-1}-1}^{i-1}\right), r \not \subset \sigma_{i, 0}\left(a_{0}^{i}\right)\right.$, and $\left.|r|=3\right\}$, and then we know that $|R| \leq\left(\begin{array}{l}4 \\ 2\end{array}\right) \times\left(\begin{array}{l}4 \\ 1\end{array}\right) \times 2=48$. Suppose again that there are $t_{1} l$ 's such that $\left|\left(\sigma_{i-1}\left(a_{q_{i-1}-1}^{i-1}\right) \cup \sigma_{i, 0}\left(a_{0}^{i}\right)\right) \cap\left(\sigma_{l-1}\left(a_{q_{l-1}-1}^{l-1}\right) \cup \sigma_{l}\left(a_{0}^{l}\right)\right)\right|=8$ and $t_{2} l$ 's such that $\left|\left(\sigma_{i-1}\left(a_{q_{i-1}-1}^{i-1}\right) \cup \sigma_{i, 0}\left(a_{0}^{i}\right)\right) \cap\left(\sigma_{l-1}\left(a_{q_{l-1}-1}^{l-1}\right) \cup \sigma_{l}\left(a_{0}^{l}\right)\right)\right|=7$. For the former case, just as in case (b.2) of Theorem 2.4, we can prove that there are at most $3(m-1)+\left(s_{j}-1\right)+1=$ $3 m+s_{j}-3 \sigma \in \Gamma_{i}$ such that $d\left(\sigma_{i-1}\left(a_{q_{i-1}-1}^{i-1}\right) \cup \sigma\left(a_{0}^{i}\right), \sigma_{l-1}\left(a_{q_{l-1}-1}^{l-1}\right) \cup \sigma_{l}\left(a_{0}^{l}\right)\right)<4$. Similarly, for the latter case, we can prove that there are at $\operatorname{most} \max \left\{m, s_{j}\right\}=m \sigma \in \Gamma_{i}$ such that $d\left(\sigma_{i-1}\left(a_{q_{i-1}-1}^{i-1}\right) \cup \sigma\left(a_{0}^{i}\right), \sigma_{l-1}\left(a_{q_{l-1}-1}^{l-1}\right) \cup \sigma_{l}\left(a_{0}^{l}\right)\right)<4$. Since $8 t_{1}+5 t_{2} \leq|R| \leq$ 48 , we have $t_{1} \leq|R| / 8 \leq 6$, and $t_{1}\left(3 m+s_{j}-3\right)+t_{2} m \leq t_{1}\left(3 m+s_{j}-3\right)+\left(48-8 t_{1}\right) m / 5=$ $t_{1}\left(1.4 m+s_{j}-3\right)+9.6 m \leq 6\left(1.4 m+s_{j}-3\right)+9.6 m=18 m+6 s_{j}-18$. From the assumptions that $m \geq 4$ and $s_{j} \geq 2$, we have $(m-2) m^{2} s_{j}>18 m+6 s_{j}-18$, which implies that there exists at least one permutation $\sigma_{i} \in \Gamma_{i}$ such that $\sigma_{i-1}\left(a_{q_{i-1}-1}^{i-1}\right) \cap \sigma_{i}\left(a_{0}^{i}\right)=\emptyset$ and $d\left(\sigma_{i-1}\left(a_{q_{i-1}-1}^{i-1}\right) \cup \sigma_{i}\left(a_{0}^{i}\right), \sigma_{l-1}\left(a_{q_{l-1}-1}^{l-1}\right) \cup \sigma_{l}\left(a_{0}^{l}\right)\right) \geq 4$ for any $0 \leq l<i-1$.

(b.2.2) If $x_{i} \neq \infty_{j}$ for any $i$ and $j, 1 \leq i \leq 4,1 \leq j \leq e$, then the proof is exactly the same as that for (b.2.1) except that $s_{j}$ is replaced by $m$.

Corollary 2.6. Let $m \geq 4$ and $g n \geq 16$. Assume there exists a $G D D(3,4, g n)$ of type $g^{n}$. If there exists a CSCU-GDD $(3,4,4 m)$ of type $m^{4}$, then there exists a $C S C U-G D D(3,4$, gnm $)$ of type $(\mathrm{mg})^{n}$.

Proof. In a $\operatorname{GDD}(3,4, g n)$ of type $g^{n}$, the number of blocks is $\lambda_{0}=\frac{g^{3} n(n-1)(n-2)}{24}$, the number of blocks containing one point is $\lambda_{1}=\frac{g^{2}(n-1)(n-2)}{6}$, and the number of blocks containing two distinct points is $\lambda_{2}=\frac{g(n-2)}{2}$. There exist two disjoint blocks if and only if $\lambda_{0}>\left(\begin{array}{l}4 \\ 1\end{array}\right)\left(\lambda_{1}-1\right)-\left(\begin{array}{l}4 \\ 2\end{array}\right)\left(\lambda_{2}-1\right)+1$. This inequality is satisfied provided that $g n \geq 16$. Then apply Theorem 2.5 with $e=0$.

THEOREM 2.7. There exists a CSCU-GDD $(3,4,4 g)$ of type $g^{4}$ for any $g \geq 5$.

Proof. Let $X=Z_{4} \times Z_{g}$. We build a $\operatorname{GDD}(3,4,4 g)$ of type $g^{4}$ on $X$ with the group set $\mathcal{G}=\left\{\{i\} \times Z_{g} \mid i \in Z_{4}\right\}$ and the block set $\mathcal{B}=\{\alpha(i, j, k)=\{(0, i),(1, i+$ $\left.j),(2, k),(3, k+j)\} \mid i, j, k \in Z_{g}\right\}$. Let $T(j, k)=\langle\alpha(0, j, k), \alpha(1, j, k+1), \ldots, \alpha(g-$ $1, j, k+g-1)\rangle, T_{j}=\langle T(j, j), T(j, j+1), \ldots, T(j, j-1)\rangle$, and $S=\left[T_{0}, T_{1}, \ldots, T_{g-1}\right]$. It is clear that $S=\mathcal{B}$ if we view $S$ as a block set. We will check that $S$ is in fact a CSCU.

It is easy to check that any two consecutive blocks in $S$ are disjoint and $d(\alpha(i, j, k)$, $\left.\alpha\left(i^{\prime}, j^{\prime}, k^{\prime}\right)\right) \geq 4$ for any distinct $(i, j, k)$ and $\left(i^{\prime}, j^{\prime}, k^{\prime}\right)$. Let $c_{t}$ be the union of two consecutive blocks. Then $d\left(\alpha(i, j, k), c_{t}\right) \geq 4$ for any $c_{t} \in \widehat{S}$. Thus we need only consider the distance between any two unions. We separate the unions into the 
following three types.

Type I: $c_{1}(i, j, k)=\alpha(i, j, k) \cup \alpha(i+1, j, k+1), 0 \leq i \leq g-2,0 \leq j, k \leq g-1$.

Type II: $c_{2}(j, k)=\alpha(g-1, j, k-2) \cup \alpha(0, j, k), 0 \leq j, k \leq g-1$.

Type III: $c_{3}(j)=\alpha(g-1, j-1, j-3) \cup \alpha(0, j, j), 0 \leq j \leq g-1$.

We should check that any two unions from these three types have distance more than or equal to 4 . Let $n_{q}$ be the number of points in $c_{1}(i, j, k) \cap c_{1}\left(i^{\prime}, j^{\prime}, k^{\prime}\right)$ with the first coordinate being $q$, where $q \in Z_{4}$. Then $n_{q} \leq 2$ for any $q \in Z_{4}$. If there are at least two $n_{q}$ 's of the $c_{1}(i, j, k) \cap c_{1}\left(i^{\prime}, j^{\prime}, k^{\prime}\right)$ having value no more than 1 , then $\left|c_{1}(i, j, k) \cap c_{1}\left(i^{\prime}, j^{\prime}, k^{\prime}\right)\right| \leq 6$, which means that $d\left(c_{1}(i, j, k), c_{1}\left(i^{\prime}, j^{\prime}, k^{\prime}\right)\right) \geq 4$.

Case a: Two unions from Type I, say, $c_{1}(i, j, k)=\{(0, i),(0, i+1),(1, i+j),(1, i+$ $j+1),(2, k),(2, k+1),(3, k+j),(3, k+j+1)\}$ and $c_{1}\left(i^{\prime}, j^{\prime}, k^{\prime}\right)=\left\{\left(0, i^{\prime}\right),\left(0, i^{\prime}+1\right),\left(1, i^{\prime}+\right.\right.$ $\left.\left.j^{\prime}\right),\left(1, i^{\prime}+j^{\prime}+1\right),\left(2, k^{\prime}\right),\left(2, k^{\prime}+1\right),\left(3, k^{\prime}+j^{\prime}\right),\left(3, k^{\prime}+j^{\prime}+1\right)\right\}$. We will show that $\left|c_{1}(i, j, k) \cap c_{1}\left(i^{\prime}, j^{\prime}, k^{\prime}\right)\right| \leq 6$ for any distinct $(i, j, k)$ and $\left(i^{\prime}, j^{\prime}, k^{\prime}\right)$. Note the fact that if $l \neq l^{\prime}$ and $g \geq 5$, then $\left|\{l, l+1\} \cap\left\{l^{\prime}, l^{\prime}+1\right\}\right| \leq 1$. Since each of the three parameters $\{i, j, k\}$ is related to two different first coordinates, it is easy to check that at least two of the $n_{q}$ 's have value no more than 1 . The details are listed below.

(1) When $i \neq i^{\prime}, j \neq j^{\prime}$, and $k \neq k^{\prime}$, we have $n_{0} \leq 1$ and $n_{2} \leq 1$.

(2) When $i \neq i^{\prime}, j \neq j^{\prime}$, and $k=k^{\prime}$, then $k+j \neq k^{\prime}+j^{\prime}$, so $n_{0} \leq 1$ and $n_{3} \leq 1$.

(3) When $i \neq i^{\prime}, j=j^{\prime}$, and $k \neq k^{\prime}$, we have $n_{0} \leq 1$ and $n_{2} \leq 1$.

(4) When $i \neq i^{\prime}, j=j^{\prime}$, and $k=k^{\prime}$, then $i+j \neq i^{\prime}+j^{\prime}$, so $n_{0} \leq 1$ and $n_{1} \leq 1$.

(5) When $i=i^{\prime}, j \neq j^{\prime}$, and $k \neq k^{\prime}$, then $i+j \neq i^{\prime}+j^{\prime}$, so $n_{1} \leq 1$ and $n_{2} \leq 1$.

(6) When $i=i^{\prime}, j \neq j^{\prime}$, and $k=k^{\prime}$, then $i+j \neq i^{\prime}+j^{\prime}$ and $k+j \neq k^{\prime}+j^{\prime}$, so $n_{1} \leq 1$ and $n_{3} \leq 1$.

(7) When $i=i^{\prime}, j=j^{\prime}$, and $k \neq k^{\prime}$, then $k+j \neq k^{\prime}+j^{\prime}$, so $n_{2} \leq 1$, and $n_{3} \leq 1$.

Case b: Two unions from Type I and Type II, respectively, say, $c_{1}(i, j, k)=$ $\{(0, i),(0, i+1),(1, i+j),(1, i+j+1),(2, k),(2, k+1),(3, k+j),(3, k+j+1)\}$ and $c_{2}\left(j^{\prime}, k^{\prime}\right)=\left\{(0, g-1),(0,0),\left(1, j^{\prime}-1\right),\left(1, j^{\prime}\right),\left(2, k^{\prime}-2\right),\left(2, k^{\prime}\right),\left(3, k^{\prime}+j^{\prime}-2\right),\left(3, k^{\prime}+\right.\right.$ $\left.\left.j^{\prime}\right)\right\}$. Since $0 \leq i \leq g-2$ in $c_{1}(i, j, k)$, we know that $n_{0} \leq 1$. Since $g \geq 5$, we have $\left|\{k, k+1\} \cap\left\{k^{\prime}-2, k^{\prime}\right\}\right| \leq 1$, i.e., $n_{2} \leq 1$. Then $d\left(c_{1}(i, j, k), c_{2}\left(j^{\prime}, k^{\prime}\right)\right) \geq 4$.

Case c: Two unions from Type I and Type III, respectively, say, $c_{1}(i, j, k)=$ $\{(0, i),(0, i+1),(1, i+j),(1, i+j+1),(2, k),(2, k+1),(3, k+j),(3, k+j+1)\}$ and $c_{3}\left(j^{\prime}\right)=\left\{(0, g-1),(0,0),\left(1, j^{\prime}-2\right),\left(1, j^{\prime}\right),\left(2, j^{\prime}-3\right),\left(2, j^{\prime}\right),\left(3,2 j^{\prime}-4\right),\left(3,2 j^{\prime}\right)\right\}$. Since $0 \leq i \leq g-2$ and $g \geq 5$, in a similar way, we can know that $n_{0} \leq 1$ and $n_{1} \leq 1$.

Case d: Two unions from Type II, say, $c_{2}(j, k)=\{(0, g-1),(0,0),(1, j-1),(1, j)$, $(2, k-2),(2, k),(3, k+j-2),(3, k+j)\}$ and $c_{2}\left(j^{\prime}, k^{\prime}\right)=\left\{(0, g-1),(0,0),\left(1, j^{\prime}-\right.\right.$ $\left.1),\left(1, j^{\prime}\right),\left(2, k^{\prime}-2\right),\left(2, k^{\prime}\right),\left(3, k^{\prime}+j^{\prime}-2\right),\left(3, k^{\prime}+j^{\prime}\right)\right\}$, where $(j, k)$ and $\left(j^{\prime}, k^{\prime}\right)$ are distinct. Similarly to Case a, we can show that there are at least two $n_{q}$ 's having value no more than 1 .

Case e: Two unions from Type II and Type III, respectively, say, $c_{2}(j, k)=$ $\{(0, g-1),(0,0),(1, j-1),(1, j),(2, k-2),(2, k),(3, k+j-2),(3, k+j)\}$ and $c_{3}\left(j^{\prime}\right)=$ $\left\{(0, g-1),(0,0),\left(1, j^{\prime}-2\right),\left(1, j^{\prime}\right),\left(2, j^{\prime}-3\right),\left(2, j^{\prime}\right),\left(3,2 j^{\prime}-4\right),\left(3,2 j^{\prime}\right)\right\}$. It is readily checked that at least one of the following assertions holds:

(e.1): $n_{1} \leq 1$ and $n_{2} \leq 1$

(e.2): $n_{1} \leq 1$ and $n_{3} \leq 1$.

Case f: Two unions from Type III, say, $c_{3}(j)=\{(0, g-1),(0,0),(1, j-2),(1, j)$, $(2, j-3),(2, j),(3,2 j-4),(3,2 j)\}$ and $c_{3}\left(j^{\prime}\right)=\left\{(0, g-1),(0,0),\left(1, j^{\prime}-2\right),\left(1, j^{\prime}\right),\left(2, j^{\prime}-\right.\right.$ $\left.3),\left(2, j^{\prime}\right),\left(3,2 j^{\prime}-4\right),\left(3,2 j^{\prime}\right)\right\}$, where $j \neq j^{\prime}$. Similarly to Case a, we can prove that there are at least two $n_{q}$ 's having value no more than 1 . 
3. Direct constructions. In this section, we directly construct some small CSCUs which will be used in the recursive constructions. In order to save space, we list only a few examples. The interested reader is referred to the authors or to the new results website for Handbook of Combinatorial Designs [2] maintained by Professor Jeff Dinitz of the University of Vermont for a copy of the detailed cyclic sequences of blocks.

Lemma 3.1. There exists a CSCU-CQS( $\left.g^{n}: s\right)$ for each $(g, n, s) \in\{(4,4,2),(4$, $4,4),(6,3,2),(6,3,4),(6,5,2),(6,5,4),(8,3,2),(12,3,2),(12,3,4),(12,4,2),(12,4,4)\}$.

Proof. We list only the sequence of a CSCU-CQS $\left(4^{4}: 2\right)$ on the point set $X=Z_{18}$, with the group set $\mathcal{G}=\left\{\{i, 4+i, 8+i, 12+i\} \mid i \in Z_{4}\right\}$ and the stem $S=\{16,17\}$.

$S=[\{6,7,12,17\},\{4,10,11,16\},\{12,14,15,17\},\{2,5,7,16\},\{1,6,8,10\},\{2,7,9,17\},\{5,10,15,16\}$,

$\{0,1,2,14\},\{4,5,6,10\},\{1,3,14,16\},\{0,6,9,10\},\{1,2,15,17\},\{3,6,9,16\},\{1,7,10,17\}$,

$\{9,11,14,16\},\{3,5,6,17\},\{2,4,13,14\},\{1,6,11,16\},\{3,13,14,17\},\{1,7,11,12\},\{2,8,9,14\}$,

$\{0,7,11,13\},\{2,5,12,14\},\{4,6,7,8\},\{0,3,5,15\},\{6,10,12,13\},\{1,3,4,15\},\{5,7,8,11\}$,

$\{0,2,3,12\},\{4,7,9,11\},\{3,8,13,15\},\{0,10,11,12\},\{2,5,9,15\},\{3,4,8,10\},\{0,6,12,15\}$,

$\{2,4,8,11\},\{3,9,12,15\},\{1,2,7,13\},\{4,8,14,15\},\{1,3,6,13\},\{2,4,9,10\},\{0,7,12,14\}$,

$\{5,6,9,11\},\{1,10,13,15\},\{3,5,9,14\},\{0,2,10,13\},\{5,6,8,14\},\{0,1,3,7\},\{2,6,8,13\}$,

$\{5,7,9,10\},\{1,11,13,14\},\{2,5,8,10\},\{3,11,12,13\},\{0,2,5,6\},\{3,7,8,9\},\{1,4,10,14\}$,

$\{2,6,9,12\},\{3,4,5,11\},\{1,2,10,12\},\{0,3,9,11\},\{1,2,4,6\},\{8,10,13,14\},\{3,5,7,12\}$,

$\{0,6,13,14\},\{1,3,8,11\},\{0,5,10,14\},\{3,4,7,13\},\{9,10,12,14\},\{4,5,7,15\},\{1,6,12,14\}$,

$\{8,9,11,15\},\{0,3,4,6\},\{7,12,13,15\},\{4,6,9,14\},\{5,11,12,15\},\{0,3,8,14\},\{4,11,13,15\}$,

$\{0,7,8,10\},\{1,9,14,15\},\{0,2,4,7\},\{3,6,8,12\},\{0,1,11,15\},\{2,7,8,12\},\{0,4,11,14\}$,

$\{1,7,8,15\},\{3,4,12,14\},\{0,7,9,15\},\{4,6,11,12\},\{1,3,9,10\},\{8,11,12,14\},\{1,2,3,5\}$,

$\{0,4,10,15\},\{1,6,7,9\},\{0,2,8,15\},\{4,7,10,12\},\{1,2,9,11\},\{8,10,12,15\},\{2,3,9,13\}$,

$\{0,6,8,11\},\{5,13,14,15\},\{2,3,6,7\},\{1,5,10,11\},\{0,4,9,13\},\{10,11,14,15\},\{5,6,7,13\}$,

$\{2,4,12,15\},\{9,10,11,13\},\{1,5,8,12\},\{7,9,13,14\},\{0,1,4,5\},\{2,6,11,15\},\{3,7,10,14\}$,

$\{2,5,11,13\},\{1,4,9,12\},\{2,3,14,15\},\{0,5,8,13\},\{2,7,10,15\},\{0,1,12,13\},\{6,7,14,15\}$,

$\{4,5,8,9\},\{2,7,11,14\},\{1,5,6,15\},\{8,9,12,13\},\{3,6,11,14\},\{4,5,12,13\},\{6,7,10,11\}$,

$\{0,1,8,9\},\{3,6,10,15\},\{1,5,7,14\},\{6,9,13,15\},\{2,3,10,11\},\{4,5,14,16\},\{2,12,13,17\}$,

$\{0,1,10,16\},\{2,4,5,17\},\{8,9,10,16\},\{0,1,6,17\},\{3,5,10,13\},\{6,8,9,17\},\{12,13,14,16\}$,

$\{5,8,15,17\},\{1,4,7,16\},\{0,5,9,12\},\{1,4,8,13\},\{5,10,12,17\},\{0,2,9,16\},\{4,10,13,17\}$,

$\{1,2,8,16\},\{0,9,14,17\},\{4,6,13,16\},\{1,8,14,17\},\{5,6,12,16\},\{0,13,15,17\},\{7,9,12,16\}$,

$\{1,4,11,17\},\{0,3,13,16\},\{9,11,12,17\},\{3,5,8,16\},\{0,2,11,17\},\{1,12,15,16\},\{3,4,9,17\}$,

$\{0,6,7,16\},\{1,3,12,17\},\{0,5,11,16\},\{7,8,13,17\},\{4,9,15,16\},\{0,5,7,17\},\{6,8,15,16\}$,

$\{0,3,10,17\},\{8,11,13,16\},\{4,6,15,17\},\{7,8,14,16\},\{6,11,13,17\},\{3,10,12,16\},\{4,7,14,17\}$,

$\{2,11,12,16\},\{9,10,15,17\},\{2,3,4,16\},\{8,10,11,17\},\{0,14,15,16\},\{2,3,8,17\},\{7,10,13,16\}$,

$\{5,11,14,17\},\{2,13,15,16\}]$.

Lemma 3.2. There exists a CSCU-GDD $\left(g^{u}\right)$ for each $(g, u) \in\{(3,4),(4,4),(4,5)$, $(6,5),(6,6)\}$.

Proof. We list only two examples here. First, we list the sequence of a CSCU$\operatorname{GDD}\left(3^{4}\right)$ on the point set $X=Z_{12}$ with the group set $\mathcal{G}=\left\{\{i, 4+i, 8+i\} \mid i \in Z_{4}\right\}$.

$S=[\{0,1,2,3\},\{4,5,6,7\},\{8,9,10,11\},\{0,1,6,7\},\{4,5,10,11\},\{8,9,2,3\},\{0,1,10,11\}$, $\{4,5,2,3\},\{8,9,6,7\},\{0,5,10,3\},\{4,9,2,7\},\{8,1,6,11\},\{0,5,2,7\},\{4,9,6,11\}$, $\{8,1,10,3\},\{0,5,6,11\},\{4,9,10,3\},\{8,1,2,7\},\{0,9,6,3\},\{4,1,10,7\},\{8,5,2,11\}$, $\{0,9,10,7\},\{4,1,2,11\},\{8,5,6,3\},\{0,9,2,11\},\{4,1,6,3\},\{8,5,10,7\}]$. 
Next, we list the sequence of a CSCU-GDD $\left(4^{4}\right)$ on the point set $X=Z_{16}$ with the group set $\mathcal{G}=\left\{\{i, 4+i, 8+i, 12+i\} \mid i \in Z_{4}\right\}$.

$S=[\{0,1,2,3\},\{4,5,6,7\},\{8,9,10,11\},\{12,13,14,15\},\{0,1,6,7\},\{4,5,10,11\},\{8,9,14,15\}$, $\{2,3,12,13\},\{0,1,10,11\},\{4,5,14,15\},\{2,3,8,9\},\{6,7,12,13\},\{2,3,4,5\},\{0,1,14,15\}$, $\{6,7,8,9\},\{10,11,12,13\},\{0,2,5,7\},\{4,6,9,11\},\{8,10,13,15\},\{1,3,12,14\},\{0,5,6,11\}$, $\{4,9,10,15\},\{3,8,13,14\},\{1,2,7,12\},\{0,5,10,15\},\{3,4,9,14\},\{2,7,8,13\},\{1,6,11,12\}$, $\{2,4,7,9\},\{6,8,11,13\},\{1,10,12,15\},\{0,3,5,14\},\{4,6,13,15\},\{0,2,9,11\},\{1,3,8,10\}$, $\{5,7,12,14\},\{0,6,9,15\},\{3,4,10,13\},\{1,7,8,14\},\{2,5,11,12\},\{0,3,9,10\},\{4,7,13,14\}$, $\{1,2,8,11\},\{5,6,12,15\},\{2,4,11,13\},\{1,6,8,15\},\{3,5,10,12\},\{0,7,9,14\},\{1,3,4,6\}$, $\{0,2,13,15\},\{5,7,8,10\},\{9,11,12,14\},\{0,3,6,13\},\{1,4,7,10\},\{5,8,11,14\},\{0,7,10,13\}$, $\{5,9,12,15\},\{1,4,11,14\},\{2,5,8,15\},\{3,6,9,12\},\{1,2,4,15\},\{0,11,13,14\},\{3,5,6,8\}$, $\{7,9,10,12\}]$.

LEmma 3.3. There exists a CSCU-HSQS $(v: s)$ for each $(v, s) \in\{(16,4),(20,8)$, $(22,10),(26,10)\}$.

Proof. Here we list only the sequence of a CSCU-HSQS(16:4) on the point set $X=Z_{16}$ with the hole set $\{0,1,2,3\}$.

$S=[\{3,4,11,12\},\{0,1,6,7\},\{8,9,10,11\},\{0,1,4,5\},\{2,3,6,7\},\{8,9,12,13\},\{0,2,4,6\}$, $\{8,9,14,15\},\{0,2,5,7\},\{8,10,12,14\},\{0,3,4,7\},\{8,10,13,15\},\{0,3,5,6\},\{8,11,12,15\}$, $\{4,5,6,7\},\{8,11,13,14\},\{2,3,4,5\},\{12,13,14,15\},\{1,3,5,7\},\{10,11,14,15\},\{1,3,4,6\}$, $\{10,11,12,13\},\{1,2,5,6\},\{9,11,13,15\},\{1,2,4,7\},\{9,11,12,14\},\{0,1,10,15\},\{2,7,8,9\}$, $\{0,1,11,14\},\{2,7,10,15\},\{0,1,12,13\},\{2,7,11,14\},\{9,10,12,15\},\{3,6,11,14\},\{0,1,8,9\}$, $\{3,6,10,15\},\{2,7,12,13\},\{4,5,10,15\},\{3,6,8,9\},\{0,2,12,15\},\{4,5,8,9\},\{3,6,12,13\}$, $\{4,5,11,14\},\{1,3,12,15\},\{9,10,13,14\},\{5,6,12,15\},\{0,2,8,10\},\{4,5,12,13\},\{0,2,9,11\}$, $\{1,3,8,10\},\{0,2,13,14\},\{1,3,9,11\},\{4,7,8,10\},\{1,3,13,14\},\{4,7,9,11\},\{5,6,8,10\}$, $\{2,4,9,13\},\{0,3,8,11\},\{4,7,13,14\},\{1,5,10,12\},\{0,3,9,13\},\{4,7,12,15\},\{5,6,13,14\}$, $\{0,3,10,12\},\{2,4,8,11\},\{0,3,14,15\},\{5,6,9,11\},\{2,4,10,12\},\{1,5,9,13\},\{0,4,8,12\}$, $\{1,5,14,15\},\{6,7,10,12\},\{2,4,14,15\},\{1,5,8,11\},\{6,7,9,13\},\{0,4,10,14\},\{6,7,8,11\}$, $\{0,4,9,15\},\{2,6,11,13\},\{3,5,8,12\},\{0,4,11,13\},\{6,7,14,15\},\{3,5,11,13\},\{2,6,10,14\}$, $\{3,5,9,15\},\{1,7,8,12\},\{3,5,10,14\},\{2,6,8,12\},\{1,7,10,14\},\{2,6,9,15\},\{0,5,8,13\}$, $\{1,7,9,15\},\{0,5,12,14\},\{4,6,11,15\},\{0,5,9,10\},\{1,7,11,13\},\{4,6,12,14\},\{3,7,8,13\}$, $\{0,5,11,15\},\{4,6,8,13\},\{3,7,11,15\},\{4,6,9,10\},\{3,7,12,14\},\{1,2,8,13\},\{3,7,9,10\}$, $\{1,2,12,14\},\{0,6,13,15\},\{1,2,9,10\},\{0,6,8,14\},\{1,2,11,15\},\{0,6,9,12\},\{5,7,8,14\}$, $\{1,4,13,15\},\{0,6,10,11\},\{5,7,13,15\},\{1,4,9,12\},\{5,7,10,11\},\{1,4,8,14\},\{5,7,9,12\}$, $\{2,3,8,14\},\{1,4,10,11\},\{2,3,13,15\},\{0,7,9,14\},\{2,3,10,11\},\{0,7,8,15\},\{1,6,9,14\}$, $\{0,7,11,12\},\{1,6,8,15\},\{2,5,10,13\},\{3,4,8,15\},\{1,6,10,13\},\{2,3,9,12\},\{0,7,10,13\}$, $\{2,5,9,14\},\{3,4,10,13\},\{2,5,11,12\},\{3,4,9,14\},\{1,6,11,12\},\{2,5,8,15\}]$.

Lemma 3.4. There exists a $B S C U(v)$ for each $v \in\{20,22,26,34,38\}$.

Proof. Here we show only the existence of a BSCU(20). Take $X=Z_{19} \cup\{\infty\}$ as the point set. Let

$$
\begin{aligned}
A= & {[\{0,4,5,6\},\{2,7,12,14\},\{1,3,4,9\},\{0,8,14,17\},\{\infty, 1,7,9\},\{5,11,18,2\},} \\
& \{0,1,3,7\},\{2,6,11,17\},\{\infty, 1,10,13\},\{14,16,18,2\},\{7,15,0,6\},\{1,8,9,12\}, \\
& \{5,6,13,15\},\{1,2,12,17\},\{\infty, 8,9,13\}],
\end{aligned}
$$

and $S=[A, A+1, A+2, \ldots, A+18]$, where additions are taken modulo 19. Then $S$ is the required $\operatorname{BSCU}(20)$. 
4. Results obtained by recursion. First, we list some known results on 3designs. A $t$-wise balanced design (or $t$-BD) of order $v$ and block sizes from $K$, denoted by $\mathrm{S}(t, K, v)$, is a pair $(X, \mathcal{B})$, where $X$ is a set of $v$ elements (called points), $\mathcal{B}$ is a collection of subsets (called blocks) of $X$, each of cardinality from $K$, such that every $t$-subset of $X$ is contained in a unique block of $\mathcal{B}$. The set of all positive integers $v$ such that an $\mathrm{S}(t, K, v)$ exists is denoted by $B_{t}(K)$.

TheOrem 4.1. (see [13]). There exists a $\operatorname{CQS}\left(6^{n}: 0\right)$ for any positive integer $n$.

TheOREM 4.2. (see $[5]) . B_{3}(\{4\})=\{v>0 \mid v \equiv 2,4(\bmod 6)\}$.

Theorem 4.3. (see [11]). $B_{3}(\{4,5,6\})=\{v>0 \mid v \equiv 0,1,2(\bmod 4)$ and $v \neq$ $9,13\}$.

Lemma 4.4. (see [15]). There exists a $\operatorname{BSCU}(v)$ for $v \in\{14,16,32,46,56\}$.

LEMMA 4.5. If there exists a CSCU-GDD $\left(g^{n}\right)$, then there exists a CSCU-GDD $\left((m g)^{n}\right)$ for any integer $m \geq 3$.

Proof. Combining Lemma 3.2 with Theorem 2.7, we know that there exists a CSCU-GDD $\left(m^{4}\right)$ for any $m \geq 3$.

Let $S=\left[b_{0}, \ldots, b_{q-1}\right]$ be the CSCU-GDD $\left(g^{n}\right)$. For any $b_{i} \in S$, there is a CSCU$\operatorname{GDD}\left(m^{4}\right)$, denoted $S_{i}$, on the point set $b_{i} \times I_{m}$ for any integer $m \geq 3$. Let $S^{\prime}=$ $\left[S_{0}, \ldots, S_{q-1}\right]$. Then it is easy to check that $S^{\prime}$ is a $\operatorname{CSCU-GDD}\left((m g)^{n}\right)$.

LEMMA 4.6. There exists a CSCU-CQS $\left(12^{n}: s\right)$ for each $s \in\{8,10\}$ and $n \geq 4$.

Proof. For each $n \equiv 0,1(\bmod 3)$ and $n \geq 4$, there exists an $S(3,4,2 n+2)$ by Theorem 4.2. Deleting two points from this 3 -BD yields a $2-\mathrm{FG}(3,(\{3\},\{3\},\{4\}), 2 n)$ of type $2^{n}$. By counting the numbers of blocks in the $S(3,4,2 n+2)$ containing $t$, where $t=0,1,2$ common points, we can know that in the $2-\mathrm{FG}(3,(\{3\},\{3\},\{4\}), 2 n)$ of type $2^{n}$, when $n \geq 4$, there exist two disjoint blocks with one of size 4 and the other of size 3. For each $s \in\{8,10\}$, applying Theorem 2.4 with a CSCU-CQS $\left(6^{3}: s-6\right)$ and a CSCU-GDD $\left(6^{4}\right)$, we obtain a CSCU-CQS $\left(12^{n}: s\right)$. Here, the ingredient designs come from Theorem 2.7 and Lemma 3.1.

For any $n \equiv 2(\bmod 3)$ and $n \geq 5$, there is a $\operatorname{CQS}\left(6^{\frac{n+1}{3}}: 0\right)$ by Theorem 4.1. For $n=5,8,11$, it can be checked from the detailed construction in [13] for each of these $\operatorname{CQS}\left(6^{\frac{n+1}{3}}: 0\right)$ that there exist two disjoint blocks $a$ and $b$ intersecting two groups, say, $g_{1}$ and $g_{2}$, in two points, respectively. So there are two points $y \in g_{1}$ and $z \in g_{2}$ not covered by $a$ and $b$. Choose $x \in a \cap g_{2}$ and delete $x, y$. Then we obtain a 2-FG $(3,(\{3,5\},\{3,5\},\{4,6\}), 10)$ of type $2^{n}$ with two disjoint blocks $a \backslash\{x\} \in \mathcal{B}_{1}$ and $b \in \mathcal{T}$. For $n \geq 14$, let $x, y$ be two points from different groups $g_{x}, g_{y}$, respectively, and $g$ be a group disjoint from a block containing $x, y$. By deleting $x$ and $y$, we obtain a $2-\mathrm{FG}(3,(\{3,5\},\{3,5\},\{4,6\}), 2 n)$ of type $2^{n}$ with two disjoint blocks $g_{x} \backslash\{x\} \in \mathcal{B}_{1}$ and $g \in \mathcal{T}$. Then for each $s \in\{8,10\}$, by applying Theorem 2.4 with a CSCU$\operatorname{CQS}\left(6^{3}: s-6\right)$, a CSCU-CQS $\left(6^{5}: s-6\right)$, a CSCU-GDD $\left(6^{4}\right)$, and a CSCU-GDD $\left(6^{6}\right)$, we obtain a CSCU-CQS $\left(12^{n}: s\right)$, where the ingredient designs come from Theorem 2.7 and Lemmas 3.1 and 3.2 .

LEMMA 4.7. There exists a BSCU(v) for each $v \equiv 8,10(\bmod 12)$ and $v \geq 12$.

Proof. For each $v \in\{20,22,32,34,46\}$, there is a BSCU $(v)$ by Lemmas 3.4 and 4.4 . For $v=44$, there is a $\operatorname{BSCU}(v)$ by applying Theorem 1.1.(1) with a $\operatorname{BSCU}(22)$.

For each $v \equiv 8,10(\bmod 12)$ and $v \geq 56$, there is a CSCU-CQS $\left(12^{n}: s\right)$ where $v=12 n+s, n \geq 4$, and $s \in\{8,10\}$ by Lemma 4.6 . Then by applying Theorem 2.3 with a CSCU-HSQS(12+s $: s)$, we obtain a CSCU-HSQS(12n+s:12+s), and furthermore, by applying Theorem 2.2 with a BSCU $(12+s)$, we obtain a BSCU $(12 n+s)$, where the ingredient CSCU-HSQS comes from Lemma 3.3.

LEMmA 4.8. There exists a CSCU-GDD $\left(12^{u}\right)$ for each $u \in\{5,6\}$. 
Proof. From Lemma 3.2, we know that there exists a CSCU-GDD $\left(4^{5}\right)$. Applying Lemma 4.5 with $m=3$, we obtain a CSCU-GDD $\left(12^{5}\right)$.

From Theorem 2.1, we know that there exists a $\operatorname{GDD}\left(3^{6}\right)$. Applying Corollary 2.6 with a CSCU-GDD $\left(4^{4}\right)$ from Lemma 3.2 , we obtain a CSCU-GDD $\left(12^{6}\right)$.

LEMma 4.9. There exists a CSCU-CQS $\left(12^{n}: s\right)$ for each $n \in\{5,8\}$ and $s \in$ $\{2,4\}$.

Proof. For each $n \in\{5,8\}$, there is an $\mathrm{S}(3,5,3 n+2)$ in [6]. Deleting two points gives a $2-\mathrm{FG}(3,(\{4\},\{4\},\{5\}), 3 n)$ of type $3^{n}$, which is also a $1-\mathrm{FG}(3,(\{4\},\{4,5\}), 3 n)$ of type $3^{n}$. By counting the numbers of blocks in the $S(3,5,3 n+2)$ containing $t$, where $t=0,1,2$ common points, we can know that in the 2 -FG $(3,(\{4\},\{4\},\{5\}), 3 n)$ of type $3^{n}$, when $n=5,8$, there exist two disjoint blocks with one of size 5 and the other of size 4 . For each $s \in\{2,4\}$, by applying Theorem 2.5 with a CSCU-CQS $\left(4^{4}: s\right)$, a CSCU-GDD $\left(4^{4}\right)$, and a CSCU-GDD $\left(4^{5}\right)$, which come from Lemmas 3.1 and 3.2 , we obtain a CSCU-CQS $\left(12^{n}: s\right)$.

LEMma 4.10. There exists a CSCU-CQS(12 $: s)$ for $s \in\{2,4\}$ and $n \equiv 0,1,3$ $(\bmod 4), n \geq 7, n \neq 8,12$.

Proof. For each $n \equiv 0,1,3(\bmod 4), n \geq 7$, and $n \neq 8,12$, there exists an $S(3,\{4,5,6\}, n+1)(X, \mathcal{B})$ by Theorem 4.3. Let $x, y$ be two points of $X$, and $b_{1}, b_{2}, \ldots, b_{w}$ be the blocks in $\mathcal{B}$ containing both $x$ and $y$. Then $\left\{b_{1} \backslash\{x, y\}, b_{2} \backslash\right.$ $\left.\{x, y\}, \ldots, b_{w} \backslash\{x, y\}\right\}$ is a partition of $X \backslash\{x, y\}$, and $2 \leq\left|b_{i} \backslash\{x, y\}\right| \leq 4$ for $i=1,2, \ldots, w$. Let $u \in b_{1} \backslash\{x, y\}, v \in b_{2} \backslash\{x, y\}$, and $b$ be a block containing both $u$ and $v$. If $w \geq 7$, which would happen if $n \geq 27$, then there must exist one $b_{i} \backslash\{x, y\}$, say, $i=i_{0}$, which is disjoint with $b$. Deleting $u$ from this 3 -BD yields a 1 -FG $(3,(\{3,4,5\},\{4,5,6\}), n)$ of type $1^{n}$ with two disjoint blocks $b \backslash\{u\} \in \mathcal{B}_{1}$ and $b_{i_{0}} \in \mathcal{T}$. For each $n \equiv 1,3(\bmod 6)$, there exists an $S(3,4, n+1)$ by Theorem 4.2 . By counting the numbers of blocks in the $S(3,4, n+1)$ containing $t$, where $t=0,1,2$ common points, we can know that there exist two disjoint blocks $b, b^{\prime}$ when $n \geq 7$. Deleting one point $x \in b$ from this 3 -BD yields a $1-\mathrm{FG}(3,(\{3\},\{4\}), n)$ of type $1^{n}$ with two disjoint blocks $b \backslash\{x\} \in \mathcal{B}_{1}$ and $b^{\prime} \in \mathcal{T}$. For $n=16$, there exists an $\mathrm{S}(3,5,17)$ from [6]. By the same method as above, we know that there exists a $1-\mathrm{FG}(3,(\{4\},\{5\}), n)$ of type $1^{n}$ with two disjoint blocks, one in $\mathcal{B}_{1}$ and the other in $\mathcal{T}$. For $n=20,24$, there exist an $\mathrm{S}(3,6,22)$ and an $\mathrm{S}(3,6,26)$ from [6]. In a similar fashion, we can prove the existence of two disjoint blocks in each of these two Steiner systems. Deleting two points from one of these two disjoint blocks yields a 1-FG $(3,(\{4,5\},\{5,6\}), n)$ of type $1^{n}$ with two disjoint blocks, one in $\mathcal{B}_{1}$ and the other in $\mathcal{T}$. For $n=11,17,23$, just as in the proof of Lemma 4.6, we can know that there exist two disjoint blocks in the $\operatorname{CQS}\left(6^{\frac{n+1}{6}}: 0\right)$. Deleting one point from one of these two disjoint blocks yields a 1$\mathrm{FG}(3,(\{3,5\},\{4,6\}), n)$ of type $1^{n}$ with two disjoint blocks, one in $\mathcal{B}_{1}$ and the other in $\mathcal{T}$. Now for each $s \in\{2,4\}$, by applying Theorem 2.4 with a CSCU-CQS $\left(12^{h}: s\right)$ and a CSCU-GDD $\left(12^{h+1}\right)$ for each $h \in\{3,4,5\}$, we obtain a CSCU-CQS $\left(12^{n}: s\right)$. Here, the ingredient designs come from Theorem 2.7 and Lemmas 3.1, 4.8, and 4.9.

Lemma 4.11. There exists a BSCU $(12 n+s)$ for $s \in\{2,4\}, n \equiv 0,1,3(\bmod 4)$, $n \geq 4$, and $n \neq 12$.

Proof. For each $n \equiv 0,1,3(\bmod 4), n \geq 4$, and $n \neq 12$, there exists a CSCUCQS $\left(12^{n}: s\right)$ for $s \in\{2,4\}$ by Lemmas $3.1,4.9$, and 4.10. Then by applying Theorem 2.3 with a CSCU-HSQS $(12+s: s)$ and Theorem 2.2 with a $\operatorname{BSCU}(12+s)$, we obtain a $\operatorname{BSCU}(12 n+s)$. Here, the ingredient designs come from Theorem 1.1 and Lemmas 3.3 and 4.4, where the BSCU(14) in Theorem 1.1 is actually a CSCU$\operatorname{HSQS}(12+2: 2)$. 
Lemma 4.12. There exists a BSCU(48n+26) for any $n \geq 0$.

Proof. A BSCU(26) was shown in Lemma 3.4. For each integer $n \geq 1$, as was shown in the proof of Lemma 4.6 , there exists a $2-\mathrm{FG}(3,(\{3\},\{3\},\{4\}), 2(3 n+1))$ of type $2^{3 n+1}$ with two disjoint blocks, one being of size 4 and the other of size 3 . Applying Theorem 2.4 with a CSCU-CQS $\left(8^{3}: 2\right)$ and a $\operatorname{CSCU}-\operatorname{GDD}\left(8^{4}\right)$, we obtain a CSCU-CQS $\left(16^{3 n+1}: 10\right)$. Then by applying Theorem 2.3 with a CSCU-HSQS $(26: 10)$ and Theorem 2.2 with a BSCU(26), we obtain a BSCU $(48 n+26)$. Here, the ingredient designs come from Theorem 2.7 and Lemmas 3.1, 3.3, and 3.4.

Lemma 4.13. There exists a BSCU(12n+s) for $n \in\{1,3,12\}$ and $s \in\{2,4\}$.

Proof. For each $v \in\{14,16,38\}$, there is a BSCU $(v)$ by Lemmas 3.4 and 4.4. For each $v \in\{40,148\}$, there is a $\operatorname{BSCU}(v)$ by applying Theorem 1.1.(1) with a BSCU $(u)$ for $u \in\{20,74\}$ in Lemmas 3.4 and 4.12 , respectively.

For $v=146$, there exists an $\mathrm{S}(3,6,26)$ in [6]. Deleting two points gives a 2$\mathrm{FG}(3,(\{5\},\{5\},\{6\}), 24)$ of type $4^{6}$, which is also a $1-\mathrm{FG}(3,(\{5\},\{5,6\}), 24)$ of type $4^{6}$. It can be easily shown that this $2-\mathrm{FG}(3,(\{5\},\{5\},\{6\}), 24)$ has two disjoint blocks with one of size 6 and the other of size 5. Applying Theorem 2.4 with a CSCU-CQS $\left(6^{5}: 2\right)$, a CSCU-GDD $\left(6^{5}\right)$, and a CSCU-GDD $\left(6^{6}\right)$, we obtain a CSCUCQS $\left(24^{6}: 2\right)$. Then applying Theorem 2.3 with a CSCU-HSQS $(26: 2)$ and Theorem 2.2 with a $\mathrm{BSCU}(26)$, we obtain a $\mathrm{BSCU}(146)$. Here, the ingredient designs come from Theorem 1.1 and Lemmas 3.1 and 3.2 , where the BSCU(26) in Theorem 1.1 is actually a CSCU-HSQS $(26: 2)$.

Lemma 4.14. There exists a BSCU(v) for $v \equiv 28(\bmod 48)$.

Proof. Combining Lemmas 4.11 and 4.13, we have the fact that there exists a $\operatorname{BSCU}(12 n+2)$ for each $n \equiv 1(\bmod 2)$. Then apply Theorem 1.1.(1).

5. Concluding remarks. Combining Lemmas 4.7 and $4.11-4.14$, we have the following conclusion.

THEOREM 5.1. The necessary conditions for the existence of a BSCU(v), namely, $v \equiv 2,4(\bmod 6)$ and $v \geq 4$, are also sufficient, with two exceptions $v=8,10$.

Acknowledgments. The authors thank the two anonymous referees for their helpful comments and Professor Jeff Dinitz of the University of Vermont for posting our useful small CSCUs on the new results website for Handbook of Combinatorial Designs, Second Edition [2].

\section{REFERENCES}

[1] C. J. Colbourn, Group testing for consecutive positives, Ann. Comb., 3 (1999), pp. 37-41.

[2] J. H. Dinitz, http://www.emba.uvm.edu/ dinitz/hcd/ge-miao-zhang.appendix.pdf, 2009.

[3] R. Dorfman, The detection of defective members of large populations, Ann. Math. Statist., 14 (1943), pp. 436-440.

[4] D.-Z. Du And F. K. Hwang, Combinatorial Group Testing and Its Applications, World Scientific, Singapore, 1993.

[5] H. Hanani, On quadruple systems, Canad. J. Math., 12 (1960), pp. 145-157.

[6] H. Hanani, A class of three-designs, J. Combin. Theory Ser. A, 26 (1979), pp. 1-19.

[7] A. Hartman, Tripling quadruple systems, Ars Combin., 10 (1980), pp. 255-309.

[8] A. Hartman, A general recursive construction for quadruple systems, J. Combin. Theory Ser. A, 33 (1982), pp. 121-134.

[9] A. Hartman, The fundamental construction for 3-designs, Discrete Math., 124 (1994), pp. 107132.

[10] L. Ji, On the $3 B D$ closed set $B_{3}(\{4,5\})$, Discrete Math., 287 (2004), pp. 55-67.

[11] L. Ji, On the $3 B D$ closed set $B_{3}(\{4,5,6\})$, J. Combin. Designs, 12 (2004), pp. 92-102.

[12] H. LenZ, Tripling Steiner quadruple systems, Ars Combin., 20 (1985), pp. 193-202.

[13] W. H. Mills, On the covering of triples by quadruples, Congr. Numer., 10 (1974), pp. 563-581. 
[14] W. H. Mills, On the existence of H designs, Congr. Numer., 79 (1990), pp. 205-240.

[15] K. Mominara And M. Jimbo, Some constructions for block sequences of Steiner quadruple systems with error correcting consecutive unions, J. Combin. Designs, 16 (2008), pp. 152163.

[16] K. Mominara And M. Jimbo, On a cyclic sequence of a packing by triples with error correcting consecutive unions, Util. Math., to appear.

[17] M. Müller And M. Jimbo, Consecutive positive detectable matrices and group testing for consecutive positives, Discrete Math., 279 (2004), pp. 369-381.

[18] M. Müller AND M. Jimbo, Cyclic sequences of $k$-subsets with distinct consecutive unions, Discrete Math., 308 (2008), pp. 457-464.

[19] F. Sagols, L. P. Riccio, and C. J. Colbourn, Dominating error correcting codes with distance two, J. Combin. Designs, 10 (2002), pp. 294-302. 